\title{
REVISED AND EXTENDED ANALYSIS OF FIVE TIMES IONIZED XENON, Xe vi
}

\author{
M. Gallardo ${ }^{1}$, M. Raineri ${ }^{1}$, J. Reyna Almandos ${ }^{1}$, C. J. B. Pagan ${ }^{2}$, and R. A. Abrahão ${ }^{2}$ \\ ${ }^{1}$ Centro de Investigaciones Opticas (CIOp), CC 3, 1897, Gonnet, La Plata, Argentina; monicar@ ciop.unlp.edu.ar \\ ${ }^{2}$ School of Electrical and Computer Engineering, University of Campinas (UNICAMP), 13083-852, Campinas, SP, Brazil; cesarpagan@ fee.unicamp.br \\ Received 2014 June 20; accepted 2014 November 14; published 2014 December 24
}

\begin{abstract}
A capillary discharge tube was used to record the Xe spectrum in the 400-5500 $\AA$ region. A set of 243 lines of the Xe vi spectrum was observed, and 146 of them were classified for the first time. For all known lines, we calculated the weighted oscillator strengths $(g f)$ and weighted transition probabilities $(g A)$ using the configuration interaction in a relativistic Hartree-Fock approach. The energy matrix was calculated using energy parameters adjusted to fit the experimental energy levels. Core polarization effects were taken into account in our calculations. Experimental energy values and calculated lifetimes are also presented for a set of 88 levels. From these levels, 32 were classified for the first time and 33 had their values revised. Our analysis of the $5 s 5 p 5 d$ and $5 s 5 p 6 s$ configurations was extended in order to clarify discrepancies among previous works.
\end{abstract}

Key words: atomic data - line: identification - ultraviolet: general - white dwarfs

\section{INTRODUCTION}

Spectroscopic data of ionized xenon are relevant to several fields of research, such as astrophysics, plasma, laser and collision physics. Xenon is a very rare element in the cosmos, observed in chemically peculiar stars (Dworetsky et al. 2008; Cowley et al. 2010) and in planetary nebulae (Péquignot \& Baluteau 1994; Sharpee et al. 2007; Sterling et al. 2009; Otsuka et al. 2010; Garcia et al. 2012; Otsuka \& Tajitsu 2013), but difficult to see in ordinary stars and not even detected in the sun. The xenon abundance in the interstellar medium is unknown. Spectroscopic data of xenon in intermediate ionization degrees-Xe II to Xe VII-have been used to observe its abundance in cosmic objects. Recently, nine Xe vi lines were observed in the ultraviolet spectrum of the hot DO-type white dwarf RE 0503-289 (Werner et al. 2012), in the first detection of xenon in this kind of star.

The ground-state configuration of five times ionized xenon (or $\mathrm{Xe} \mathrm{VI)} \mathrm{is} 5 s^{2} 5 p$ and it belongs to the In isoelectronic sequence. The line classification for this spectrum was studied for the first time by Fawcett et al. (1961) using a Zeta toroidal plasma as the light source. However, in 1980, a new experimental work was published by Fawcett \& Bromage (1980) extending their analysis and classifying 11 transitions of the $5 s^{2} 5 p-5 s 5 p^{2}$ array for the first time. In that year, Kernahan et al. (1980) published the first beam foil spectrum of Xe VI.

Kaufman \& Sugar (1987) used a spark gap discharge to study $\mathrm{Xe} \mathrm{VI} \mathrm{in} \mathrm{the} \mathrm{National} \mathrm{Bureau} \mathrm{of} \mathrm{Standards} 10.7 \mathrm{~m}$ spectrographs, and presented very accurate wavelengths. Later, Tauheed et al. (1992) used triggered spark and beam-foil light sources to identify five Xe VI intercombination lines for the first time. Collision-based spectroscopy experiments were performed by Larsson et al. (1996) and Wang et al. (1996, 1997a, 1997b) to investigate the $5 s^{2} 4 f$ and $N \geqslant 6$ configurations of Xe vI at Uppsala University.

Sarmiento et al. (1999) performed the spectral analysis for the $5 s 5 p 5 d, 5 p^{3}$, and $5 s 5 p 6 s$ configurations of Xe vi based on experiments conducted at the CIOp's laboratory, Argentina. Their results were not in agreement with the reported data from the subsequent paper on the same subject by Churilov \& Joshi (2000). Later, Reyna Almandos et al. (2001) used the data from Sarmiento et al. (1999) to support their spectral analysis, which only partially agreed with the analysis by Churilov \& Joshi (2000), publishing weighted oscillator strengths and lifetimes for this ion. Experimental results on energy levels and transitions were critically compiled by Saloman (2004), rejecting the analysis of Sarmiento et al. (1999) and Reyna Almandos et al. (2001) in favor of the conclusions of Churilov \& Joshi (2000). An experimental and theoretical study of the radiative lifetime and oscillator strength in Xe VI, including corepolarization (CP) effects in the calculation, was performed by Biémont et al. (2005).

We present this analysis in order to continue the study of the five times ionized Xe spectrum and to clarify some discrepancies that arose from previous studies. We used experimental data of the xenon spectrum covering the wavelength range $270-7000 \AA$ for the UV-Visible region.

To support our analysis, we used data from the In I-like isoelectronic sequence for Sb III to La IX that were extensively investigated at Dr. Joshi's laboratory at Saint Francis Xavier University, Antigonish, Canada (Tauheed et al. 1998; Gayasov \& Joshi 1998, 1999; Tauheed et al. 1999, 2000; Churilov et al. 2001).

We used the atomic structure package written by R. D. Cowan, described in The Theory of Atomic Structure and Spectra, TASS (1981), for relativistic Hartree-Fock (HFR) calculations and for the least-squares adjustment of the energy parameters to fit the experimental energy levels. The adjusted parameters were used to obtain weighted oscillator strengths, weighted transition rates, and lifetimes. The effect of CP was tested for lifetime and transition probability calculations. We also revisited and extended the analysis of the $5 s 5 p 5 d$ and $5 s 5 p 6 s$ configurations in an attempt to clarify the discrepancies between previous works. A total of 32 new levels are proposed. Our work resulted in a new set of classified lines, 146 of which are observed for the first time, that helped us to understand this spectrum on a more complete and reliable basis.

\section{EXPERIMENTAL DETAILS}

The light source used in this work is a capillary discharge experimental set built at CIOp (Reyna Almandos et al. 2009), consisting of a Pyrex tube of length $100 \mathrm{~cm}$ and inner diameter $0.5 \mathrm{~cm}$, in which the gas excitation is produced by discharging 
a bank of low-inductance capacitors ranging from 20 to $280 \mathrm{nF}$, charged with voltages up to $20 \mathrm{kV}$.

The wavelength range above $2100 \AA$ was recorded on a $3.4 \mathrm{~m}$ Ebert plane-grating spectrograph, with a diffraction grating of 600 lines $\mathrm{mm}^{-1}$, blazed for $5000 \AA$. The plate factor is $5.0 \AA \mathrm{mm}^{-1}$ in the first order. The Kodak 103a-O and Kodak $103 \mathrm{a}-\mathrm{F}$ plates were used to record the spectra in the first, second, and third diffraction orders. Thorium lines from an electrodeless discharge were superimposed on the spectrograms and served as reference lines. The positions of spectral lines were determined visually by means of a rotating prism photoelectric semiautomatic Grant comparator. This measurement method allows detection of asymmetric lines, as the line contours are displayed on an oscilloscope screen. For sharp lines, the settings are reproducible to within $\pm 1 \mu \mathrm{m}$. Wavelengths of the xenon spectral lines observed with the Ebert mounting spectrograph were determined by comparing them with interferometrically measured $\mathrm{Th}^{232}$ wavelengths (Valero 1968) and with known lines of Xe II and Xe III (Hansen \& Persson 1987; Persson et al. 1988). A third-order interpolation formula was used to reduce comparator settings to wavelength values. Most of the spectral lines from this region used in the analysis were recorded in the third diffraction order. The accuracy of the thorium standard wavelength values used was on the order of $0.005 \AA$, and the determination of the overall wavelength values of lines presented in this work was estimated to be correct to $\pm 0.01 \AA$ in the region above $2100 \AA$.

In the wavelength range below $2100 \AA$ light radiation emitted axially was analyzed using a $3 \mathrm{~m}$ normal incidence vacuum spectrograph with a concave diffraction grating of 1200 lines $\mathrm{mm}^{-1}$, blazed for $1200 \AA$. The plate factor in the first order is $2.77 \AA \mathrm{mm}^{-1}$. Kodak SWR and Ilford Q plates were used to record the spectra. C, N, O (Kelly 1987), and known lines of xenon (Hansen \& Persson 1987; Persson et al. 1988) served as internal wavelength standards. The uncertainty of the wavelength below $2100 \AA$ was estimated to be $\pm 0.02 \AA$.

To distinguish between different ionization states, we studied the behavior of the spectral line intensity as a function of pressure and discharge voltage. In our xenon spectrograms (Gallardo et al. 1995), as the pressure decreases and/or voltage increases, higher ionic species tend to predominate, allowing the identification of different ionization degrees. By observing the behavior of the spectral lines and using known lines of Xe VI, we were able to distinguish the different ionic states of xenon spectra.

Energy level values derived from the observed lines were determined using an iterative procedure that takes into account the wave numbers of the lines, weighted by their estimated uncertainties. The uncertainty of the adjusted experimental energy level values was assumed to be lower than $2 \mathrm{~cm}^{-1}$.

\section{THEORY}

The method used in the present work is the same as in our previous works (Pagan et al. 2011 and Raineri et al. 2012) for adjusted Hartree-Fock + Core Polarization calculations.

The definitions and the programs used here are described in "The Theory of Atomic Structure and Spectra," TASS (1981) by R. D. Cowan.

\subsection{Atomic Structure and Transition Rates}

In the present work, we used Cowan's atomic structure package to calculate the solution for relativistic HF equations includ- ing the configuration interaction (HFR, as defined in Section 14 of Chapter 7 and Chapter 13 of TASS) for Xe vi and for the studied ions of the isoelectronic In sequence. We adjusted the values of the energy parameters, i.e., the average configuration energy $E_{\text {av }}$, the Coulomb integrals $F^{k}, G^{k}$ and $R^{k}$, and the spin-orbit parameters $\zeta_{n l}$ (see Section 8-1 of TASS), as well as the coefficients of the expansion $y_{\beta J}^{\gamma}$ (see Equation (13.1) of TASS), to fit the experimental energy levels of Xe vi by means of a least-squares calculation (see Chapter 16 of TASS). With the adjusted values, we calculated the energy, lifetime, and composition of the levels, as well as the weighted transition probability rate $g A$ and weighted oscillator strengths $g f$, as defined in Equations (14.33), (14.38), and (14.42) of TASS.

\subsection{Hartree-Fock + Core Polarization}

We included the CP effects (see, for example, Curtis 2003) by simply replacing the dipole integral:

$$
\begin{aligned}
\int_{0}^{\infty} & P_{n l}(r) r P_{n^{\prime} l^{\prime}}(r) d r \rightarrow \int_{0}^{\infty} P_{n l}(r) r \cdot\left[1-\frac{\alpha_{d}}{\left(r^{2}+r_{c}^{2}\right)^{3 / 2}}\right] \\
& \times P_{n^{\prime} l^{\prime}}(r) d r-\frac{\alpha_{d}}{r_{c}^{3}} \int_{0}^{r_{c}} P_{n l}(r) r P_{n^{\prime} l^{\prime}}(r) d r .
\end{aligned}
$$

This is the same modification used by Quinet et al. (1999, 2002) and Biémont et al. (2000a, 2000b) to correct the transition matrix elements when including $\mathrm{CP}$ effects. In our case, the radial functions were obtained from the single configuration Hartree-Fock method with relativistic corrections and no modification was performed to include CP effects in the Hamiltonian. As suggested by Migdalek \& Baylis (1978), the CP approximately describes the core-valence correlation effects. Here we took into account the electronic correlation effects by using a large number of configurations in the energy matrix and, as pointed out in TASS (page 464), by scaling down the values of the Coulomb integrals by means of a least-squares adjustment. The values of $\alpha_{d}$ for two different cores were obtained from Equation (17.42) of TASS. The cut-off radius $r_{c}$, which defines the boundaries of the atomic core, is the average radius of the outermost core orbital. A detailed description of our CP calculations is provided in Section 4.2.

\section{CALCULATIONS}

In our HFR calculations, we considered 35 odd-parity and 34 even-parity configurations. For odd configurations, we used the same set as was used by Biémont et al. (2005), that is, $5 s^{2} 4 f$, $5 s^{2} 5 p, 5 s^{2} 6 p, 5 s^{2} 7 p, 5 s^{2} 8 p, 5 s^{2} 5 f, 5 s^{2} 6 f, 5 s^{2} 7 f, 5 s^{2} 8 f$, $5 s^{2} 6 h, 5 s^{2} 7 h, 5 s^{2} 8 h, 5 s^{2} 8 k, 5 p^{2} 6 p, 5 p^{2} 4 f, 5 p^{2} 5 f, 5 s 5 p 6 s$, $5 s 5 p 5 d, 5 s 5 p 6 d, 5 s 5 p 5 g, 5 s 5 p 6 g, 5 p^{3}, 5 s 5 d 4 f, 5 s 5 d 5 f$, $5 s 6 s 4 f$, and $5 s 6 s 5 f$, to which we added the $5 p^{2} 7 p, 5 p^{2} 6 f$, $5 p^{2} 8 p, 5 p^{2} 6 h, 5 p^{2} 7 f, 5 p^{2} 7 h, 5 p^{2} 8 f, 5 p^{2} 8 h$, and $5 p^{2} 8 k$ configurations. For even configurations, we used all those that were considered by Biémont et al., except $5 s 5 d^{2}$ and $5 s 6 s^{2}$, i.e., $5 s 5 p^{2}, 5 s^{2} 6 s, 5 s^{2} 7 s, 5 s^{2} 8 s, 5 s^{2} 5 d, 5 s^{2} 6 d$, $5 s^{2} 7 d, 5 s^{2} 8 d, 5 s^{2} 5 g, 5 s^{2} 6 g, 5 s^{2} 7 g, 5 s^{2} 8 g, 5 s^{2} 7 i, 5 s^{2} 8 i$, $5 p^{2} 5 d, 5 p^{2} 6 s, 5 p^{2} 6 d, 5 s 5 p 4 f, 5 s 4 f^{2}, 5 s 5 f^{2}, 5 s 5 p 5 f$, $5 s 5 p 6 f$, and $5 s 5 p 6 p$. In that set, we added the $4 d^{9} 5 p^{4}$, $5 p^{2} 7 s, 5 p^{2} 5 g, 5 p^{2} 7 d, 5 p^{2} 8 s, 5 p^{2} 6 g, 5 p^{2} 8 d, 5 p^{2} 7 g, 5 p^{2} 7 i$, $5 p^{2} 8 g$, and $5 p^{2} 8 i$ configurations. We excluded the $5 s 5 d^{2}$ and $5 s 6 s^{2}$ configurations because their interaction with the levels of interest is very weak. We also tested but did not include the $4 d^{9} 4 f 5 s^{2} 5 d, 4 d^{9} 4 f 5 s 5 p^{2}, 4 d^{9} 5 s^{2} 5 p 5 d$, 
$4 d^{9} 5 s 5 p 5 d^{2}, 4 f 5 s 5 g, 4 f 5 s 6 d, 4 f 5 s 6 g, 4 f 5 s 7 s, 4 f 5 s 7 d$, $4 f 5 s 7 g, 4 f 5 s 7 i, 4 f 5 s 8 s, 4 f 5 s 8 d, 4 f 5 s 8 g$, $4 f 5 s 8 i, 5 s 5 d 6 p$, $5 s 5 d 6 f, 5 s 5 d 6 h, 5 s 5 p 7 s, 5 s 5 p 7 d, 5 s 5 p 7 g, 5 s 5 p 7 i, 5 s 5 p 8 s$, $5 s 5 p 8 d, 5 s 5 p 8 g$, and $5 s 5 p 8 i$ odd configurations and the $4 f 5 s 5 f, 4 f 5 s 6 p, 4 d^{9} 5 s^{2} 5 p^{2}, 4 d^{9} 5 s^{2} 5 p 5 f, 4 d^{9} 5 s^{2} 5 p 6 p$, $5 d^{3}, 5 d^{2} 5 g, 5 d^{2} 6 s, 5 d^{2} 6 d, 5 d^{2} 6 g, 5 d^{2} 7 s, 5 d^{2} 7 d, 5 d^{2} 7 g$, $5 d^{2} 7 i, 5 d^{2} 8 s, 5 d^{2} 8 d, 5 d^{2} 8 g$, and $5 d^{2} 8 i$ even configurations for the same reason. We displaced all of the configuration average energies by the same amount, $+19388 \mathrm{~cm}^{-1}$, in order to set the ground state as the zero reference energy in our ab initio Hartree-Fock calculation.

Considering only the configurations with known experimental levels, the average purity for odd levels is $78 \%$ for LS and $70 \%$ for JJ coupling. For even parity, we found $93 \%$ for LS and $91 \%$ for JJ. Based on these findings, we designate all of the levels in the LS coupling scheme.

\subsection{Least-squares Calculation}

The results of the least-squares calculation are in Table 1 for odd parity and in Table 2 for even parity. In these calculations, we took into account the set of configurations described in the previous section to fit the energy parameters to the experimental energy levels.

We observed strong configuration interactions in both parities, even between configurations that have known levels and configurations that are unknown experimentally; this is the case for the $5 s^{2} 6 f-5 p^{2} 4 f, 5 s^{2} 8 f-5 s 5 p 5 g$, and $5 s^{2} 8 h-5 s 5 p 5 g$ interactions in odd parity and $5 s^{2} 6 d-5 s 4 f 5 p, 5 s^{2} 7 d-5 s 5 p 6 p$, $5 s^{2} 6 g-5 s 5 p 5 f$, and $5 s^{2} 8 d-5 s 5 p 5 f$ in even parity.

In a study of Xe VI lifetimes, Biémont et al. (2005) note their difficulty fitting the $6 \mathrm{~d}$ experimental level in their least-squares calculation as caused by the $5 s^{2} 6 d-5 s 4 f 5 p$ interaction. We solved this problem by fixing the configuration interaction (CI) integrals $R^{3}(4 f, 5 p ; 5 s, 6 d)$ and $R^{1}(4 f, 5 p ; 6 d, 5 s)$ at $80 \%$ of their Hartree-Fock values, which produced good agreement with experimental values. To adjust the $7 f$ and $8 h$ levels to their experimental values, it was necessary to fix the $F^{2}(4 f, 5 d)$ and $F^{4}(4 f, 5 d)$ of the $5 s 4 f 5 d$ configuration, which do not have observed energy levels, to $80 \%$ of their HFR values. We linked together CI integrals for $5 s 5 p 5 d-5 s 5 d 4 f$, resulting in a final adjustment to $65 \%$ of their HFR values. At the end of the leastsquares procedure, each of the remaining CI parameters present in the tables, one at a time, was left free to vary in order to minimize the standard deviation, and then it was fixed at the value found.

In our calculations, we found a standard deviation of $149 \mathrm{~cm}^{-1}$ for odd parity and $154 \mathrm{~cm}^{-1}$ for even parity.

\subsection{Polarizability}

We made the same choices as Biémont et al. (2005) to describe the core electrons. In their paper, the polarizability was considered for two different ion cores: one for $5 s^{2} n l-5 s^{2} n^{\prime} l^{\prime}$ transitions, when the core has a $[K r] 4 d^{10} 5 s^{2}$ (Xe vII) structure, and the other for transitions that preserves the $[K r] 4 d^{10} \mathrm{Cd}$ like (Xe IX) core. Before this study, the only value known for the polarizability of the $[K r] 4 d^{10}$ core was that from Fraga \& Muszyńska (1981), that is, $\alpha=0.88 a_{0}^{3}$. In this paper, we used values for the oscillator strengths $f_{\gamma^{\prime} J^{\prime}, \gamma J}$ obtained from energy parameters adjusted to experimental energy levels, as described by Raineri et al. (2012), to calculate the values of $\alpha$ in order to include the correlation effects between external electrons. We recalculated the polarizability on the basis of a set of 13 even and 63 odd configurations for Xe IX core, extending the
Table 1

Least-squares Fitted and Initio Hartree-Fock Energy Parameters of the Odd Configurations of $\mathrm{Xe} \mathrm{VI}$

\begin{tabular}{|c|c|c|c|c|c|}
\hline Configuration & Parameter & HFR & Adjusted & Adj./HFR ${ }^{\mathrm{a}}$ & \\
\hline \multirow[t]{2}{*}{$5 s^{2} 5 p$} & $E_{\text {av }}\left(5 s^{2} 5 p\right)$ & 19388 & 17929 & $92 \%$ & \\
\hline & $\zeta_{5 p}$ & 9879 & 10647 & $108 \%$ & \\
\hline \multirow[t]{2}{*}{$5 s^{2} 4 f$} & $E_{\mathrm{av}}\left(5 s^{2} 4 f\right)$ & 200340 & 194542 & $97 \%$ & \\
\hline & $\zeta_{4 f}$ & 207 & 207 & $100 \%$ & {$[\mathrm{FIX}]$} \\
\hline \multirow[t]{3}{*}{$5 p^{3}$} & $E_{\mathrm{av}}\left(5 p^{3}\right)$ & 264034 & 256131 & $97 \%$ & \\
\hline & $F^{2}(5 p, 5 p)$ & 57356 & 43692 & $76 \%$ & \\
\hline & $\zeta_{5 p}$ & 9751 & 10398 & $107 \%$ & \\
\hline \multirow[t]{2}{*}{$5 s^{2} 6 p$} & $E_{\text {av }}\left(5 s^{2} 6 p\right)$ & 278427 & 279187 & $100 \%$ & \\
\hline & $\zeta_{6 p}$ & 3227 & 3684 & $114 \%$ & \\
\hline \multirow[t]{8}{*}{$5 s 5 p 5 d$} & $E_{\mathrm{av}}(5 s 5 p 5 d)$ & 293415 & 293213 & $100 \%$ & \\
\hline & $\zeta_{5 p}$ & 10038 & 11136 & $111 \%$ & \\
\hline & $\zeta 5 d$ & 730 & 633 & $87 \%$ & \\
\hline & $F^{2}(5 p, 5 d)$ & 46813 & 38892 & $83 \%$ & \\
\hline & $G^{1}(5 s, 5 p)$ & 75265 & 49950 & $66 \%$ & \\
\hline & $G^{2}(5 s, 5 d)$ & 35890 & 30922 & $86 \%$ & \\
\hline & $G^{1}(5 p, 5 d)$ & 54504 & 42088 & $77 \%$ & \\
\hline & $G^{3}(5 p, 5 d)$ & 34700 & 27967 & $81 \%$ & \\
\hline \multirow[t]{2}{*}{$5 s^{2} 5 f$} & $E_{\mathrm{av}}\left(5 s^{2} 5 f\right)$ & 338302 & 335997 & $99 \%$ & \\
\hline & $\zeta_{5 f}$ & 72 & 72 & $100 \%$ & [FIX] \\
\hline \multirow[t]{5}{*}{$5 s 5 p 6 s$} & $E_{\mathrm{av}}(5 s 5 p 6 s)$ & 341856 & 342615 & $100 \%$ & \\
\hline & $\zeta_{5 p}$ & 10352 & 13284 & $128 \%$ & \\
\hline & $G^{1}(5 s, 5 p)$ & 75896 & 48860 & $64 \%$ & \\
\hline & $G^{0}(5 s, 6 s)$ & 4797 & 3537 & $74 \%$ & \\
\hline & $G^{1}(5 p, 6 s)$ & 7223 & 6581 & $91 \%$ & \\
\hline \multirow[t]{2}{*}{$5 s^{2} 7 p$} & $E_{\mathrm{av}}\left(5 s^{2} 7 p\right)$ & 379882 & 380627 & $100 \%$ & \\
\hline & $\zeta_{7 p}$ & 1576 & 2000 & $127 \%$ & \\
\hline \multirow[t]{2}{*}{$5 s^{2} 6 f$} & $E_{\mathrm{av}}\left(5 s^{2} 6 f\right)$ & 408683 & 411729 & $101 \%$ & \\
\hline & $\zeta_{6 f}$ & 36 & 36 & $100 \%$ & [FIX] \\
\hline \multirow[t]{2}{*}{$5 s^{2} 8 p$} & $E_{\mathrm{av}}\left(5 s^{2} 8 p\right)$ & 432265 & 430172 & $100 \%$ & \\
\hline & $\zeta_{8 p}$ & 894 & 894 & $100 \%$ & [FIX] \\
\hline \multirow[t]{2}{*}{$5 s^{2} 6 h$} & $E_{\mathrm{av}}\left(5 s^{2} 6 h\right)$ & 435904 & 434452 & $100 \%$ & \\
\hline & $\zeta_{6 h}$ & 0.7 & 0.7 & $100 \%$ & [FIX] \\
\hline \multirow[t]{2}{*}{$5 s^{2} 7 f$} & $E_{\mathrm{av}}\left(5 s^{2} 7 f\right)$ & 448501 & 450349 & $100 \%$ & \\
\hline & $\zeta_{7 f}$ & 21 & 21 & $100 \%$ & [FIX] \\
\hline \multirow[t]{2}{*}{$5 s 5 d 4 f^{(b)}$} & $F^{2}(4 f, 5 d)$ & 35524 & 28419 & $80 \%$ & [FIX] \\
\hline & $F^{4}(4 f, 5 d)$ & 20444 & 16355 & $80 \%$ & [FIX] \\
\hline $5 s^{2} 7 h$ & $E_{\mathrm{av}}\left(5 s^{2} 7 h\right)$ & 465207 & 463678 & $100 \%$ & \\
\hline & $\zeta_{7 h}$ & 0.4 & 0.4 & $100 \%$ & [FIX] \\
\hline $5 s^{2} 8 f$ & $E_{\mathrm{av}}\left(5 s^{2} 8 f\right)$ & 473261 & 475842 & $101 \%$ & \\
\hline & $\zeta_{8 f}$ & 13 & 13 & $100 \%$ & [FIX] \\
\hline $5 s^{2} 8 h$ & $E_{\mathrm{av}}\left(5 s^{2} 8 h\right)$ & 484248 & 482538 & $100 \%$ & \\
\hline & $\zeta_{8 h}$ & 0.2 & 0.2 & $100 \%$ & [FIX] \\
\hline $5 s^{2} 8 k$ & $E_{\mathrm{av}}\left(5 s^{2} 8 k\right)$ & 484551 & 482828 & $100 \%$ & \\
\hline & $\zeta_{8 k}$ & 0.1 & 0.1 & $100 \%$ & [FIX] \\
\hline $5 s^{2} 5 p-5 p^{3}$ & $R^{1}(5 s, 5 s ; 5 p, 5 p)$ & 74737 & 70452 & $94 \%$ & [FIX] \\
\hline $5 s^{2} 5 p-5 s 5 p 5 d$ & $R^{1}(5 s, 5 p ; 5 p, 5 d)$ & 62269 & 44333 & $71 \%$ & {$[\mathrm{FIX}]$} \\
\hline $5 s^{2} 5 p-5 s 5 p 5 d$ & $R^{2}(5 s, 5 p ; 5 d, 5 p)$ & 45521 & 32409 & $71 \%$ & {$[\mathrm{FIX}]$} \\
\hline $5 p^{3}-5 s 5 p 5 d$ & $R^{1}(5 p, 5 p ; 5 s, 5 d)$ & 61909 & 44700 & $72 \%$ & [FIX] \\
\hline $5 p^{3}-5 p^{2} 4 f$ & $R^{2}(5 p, 5 p ; 4 f, 5 p)$ & -42978 & -30084 & $70 \%$ & [FIX] \\
\hline $5 p^{3}-5 p^{2} 6 p$ & $R^{0}(5 p, 5 p ; 5 p, 6 p)$ & 1822 & 1986 & $109 \%$ & [FIX] \\
\hline $5 p^{3}-5 p^{2} 6 p$ & $R^{2}(5 p, 5 p ; 5 p, 6 p)$ & 8462 & 9225 & $109 \%$ & [FIX] \\
\hline $5 p^{3}-5 p^{2} 7 p$ & $R^{0}(5 p, 5 p ; 5 p, 7 p)$ & 847 & 909 & $107 \%$ & {$[\mathrm{FIX}]$} \\
\hline $5 p^{3}-5 p^{2} 7 p$ & $R^{2}(5 p, 5 p ; 5 p, 7 p)$ & 3255 & 3545 & $109 \%$ & [FIX] \\
\hline $5 s^{2} 6 p-5 s 5 p 5 d$ & $R^{1}(5 s, 6 p ; 5 p, 5 d)$ & -4077 & -4120 & $101 \%$ & [FIX] \\
\hline $5 s^{2} 6 p-5 s 5 p 5 d$ & $R^{2}(5 s, 6 p ; 5 d, 5 p)$ & 6135 & 6201 & $101 \%$ & {$[\mathrm{FIX}]$} \\
\hline $5 s^{2} 6 p-5 p^{2} 6 p$ & $R^{1}(5 s, 5 s ; 5 p, 5 p)$ & 76383 & 82977 & $109 \%$ & [FIX] \\
\hline $5 s 5 p 5 d-5 s^{2} 5 f$ & $R^{1}(5 p, 5 d ; 5 s, 5 f)$ & 19737 & 16938 & $86 \%$ & [FIX] \\
\hline $5 s 5 p 5 d-5 s^{2} 5 f$ & $R^{2}(5 p, 5 d ; 5 f, 5 s)$ & 4185 & 3591 & $86 \%$ & [FIX] \\
\hline $5 s 5 p 5 d-5 s 5 p 6 s$ & $R^{2}(5 p, 5 d ; 5 p, 6 s)$ & -12810 & -12810 & $100 \%$ & [FIX] \\
\hline $5 s 5 p 5 d-5 s 5 p 6 s$ & $R^{1}(5 p, 5 d ; 6 s, 5 p)$ & -4667 & -4667 & $100 \%$ & [FIX] \\
\hline $5 s 5 p 5 d-5 s 5 d 4 f$ & $R^{2}(5 p, 5 d ; 4 f, 5 d)$ & -33885 & -22019 & $65 \%$ & \\
\hline $5 s 5 p 5 d-5 s 5 d 4 f$ & $R^{4}(5 p, 5 d ; 4 f, 5 d)$ & -22167 & -14404 & $65 \%$ & \\
\hline $5 s 5 p 5 d-5 s 5 d 4 f$ & $R^{1}(5 p, 5 d ; 5 d, 4 f)$ & -34268 & -22268 & $65 \%$ & \\
\hline $5 s 5 p 5 d-5 s 5 d 4 f$ & $R^{3}(5 p, 5 d ; 5 d, 4 f)$ & -23470 & -15251 & $65 \%$ & \\
\hline $5 s^{2} 7 p-5 p^{2} 7 p$ & $R^{1}(5 s, 5 s ; 5 p, 5 p)$ & 76566 & 82570 & $108 \%$ & {$[\mathrm{FIX}]$} \\
\hline
\end{tabular}

Notes.

a Parameters omitted from this table: direct and exchange integrals, and spin-orbit $\zeta$ parameters set to $100 \%$ of their HFR values; CI integrals were set to $95 \%$ of their HFR values. The HFR values for average energy parameters $E_{\text {av }}$ were displaced by $19388 \mathrm{~cm}^{-1}$ in order to set the ground state at $0 \mathrm{~cm}^{-1}$. The standard deviation for the energy adjustment was $149 \mathrm{~cm}^{-1}$.

b The $5 s 5 d 4 f$ configuration does not have experimental levels. 
Table 2

Least-squares Fitted and Ab Initio Hartree-Fock Energy Parameters of the Even Configurations of Xe vI

\begin{tabular}{|c|c|c|c|c|c|}
\hline Configuration & Parameter & HFR & Adjusted & Adj./HFR ${ }^{\mathrm{a}}$ & \\
\hline \multirow[t]{5}{*}{$5 s 5 p^{2}$} & $E_{\mathrm{av}}\left(5 s 5 p^{2}\right)$ & 130857 & 129589 & $99 \%$ & \\
\hline & $F^{2}(5 p, 5 p)$ & 57354 & 46737 & $81 \%$ & \\
\hline & $\alpha$ & & 74 & [FIX] & \\
\hline & $\zeta_{5 p}$ & 9812 & 10645 & $108 \%$ & \\
\hline & $G^{1}(5 s, 5 p)$ & 74708 & 55590 & $74 \%$ & \\
\hline \multirow[t]{2}{*}{$5 s^{2} 5 d$} & $E_{\mathrm{av}}\left(5 s^{2} 5 d\right)$ & 189215 & 189396 & $100 \%$ & \\
\hline & $\zeta_{5 d}$ & 712 & 927 & $130 \%$ & \\
\hline $5 s^{2} 6 s$ & $E_{\mathrm{av}}\left(5 s^{2} 6 s\right)$ & 234023 & 233401 & $100 \%$ & \\
\hline \multirow[t]{2}{*}{$5 s^{2} 6 d$} & $E_{\mathrm{av}}\left(5 s^{2} 6 d\right)$ & 341343 & 342998 & $100 \%$ & \\
\hline & $\zeta_{6 d}$ & 298 & 358 & $120 \%$ & [FIX] \\
\hline $5 s^{2} 7 s$ & $E_{\mathrm{av}}\left(5 s^{2} 7 s\right)$ & 358659 & 359722 & $100 \%$ & \\
\hline \multirow[t]{2}{*}{$5 s^{2} 5 g$} & $E_{\mathrm{av}}\left(5 s^{2} 5 g\right)$ & 384952 & 384011 & $100 \%$ & \\
\hline & $\zeta_{5 g}$ & 1 & 1 & $100 \%$ & {$[\mathrm{FIX}]$} \\
\hline \multirow[t]{2}{*}{$5 s^{2} 7 d$} & $E_{\mathrm{av}}\left(5 s^{2} 7 d\right)$ & 411658 & 413161 & $100 \%$ & \\
\hline & $\zeta_{7 d}$ & 158 & 158 & $100 \%$ & {$[\mathrm{FIX}]$} \\
\hline \multirow[t]{2}{*}{$5 p^{2} 5 d^{\mathrm{b}}$} & $E_{\mathrm{av}}\left(5 p^{2} 5 d\right)$ & 419414 & 417614 & $100 \%$ & [FIX] \\
\hline & $\zeta_{5 p}$ & 9966 & 10839 & $109 \%$ & {$[\mathrm{FIX}]$} \\
\hline $5 s^{2} 8 s$ & $E_{\mathrm{av}}\left(5 s^{2} 8 s\right)$ & 420404 & 420979 & $100 \%$ & \\
\hline \multirow[t]{2}{*}{$5 s^{2} 6 g$} & $E_{\mathrm{av}}\left(5 s^{2} 6 g\right)$ & 434641 & 433212 & $100 \%$ & \\
\hline & $\zeta_{6 g}$ & 1 & 1 & $100 \%$ & {$[\mathrm{FIX}]$} \\
\hline \multirow[t]{2}{*}{$5 s^{2} 8 d$} & $E_{\mathrm{av}}\left(5 s^{2} 8 d\right)$ & 450720 & 449438 & $100 \%$ & [FIX] \\
\hline & $\zeta_{8 d}$ & 95 & 95 & $100 \%$ & {$[\mathrm{FIX}]$} \\
\hline \multirow[t]{2}{*}{$5 s^{2} 7 g$} & $E_{\mathrm{av}}\left(5 s^{2} 7 g\right)$ & 464406 & 465775 & $100 \%$ & \\
\hline & $\zeta_{7 g}$ & 1 & 1 & $100 \%$ & {$[\mathrm{FIX}]$} \\
\hline \multirow[t]{2}{*}{$5 s^{2} 7 i$} & $E_{\mathrm{av}}\left(5 s^{2} 7 i\right)$ & 465559 & 464427 & $100 \%$ & \\
\hline & $\zeta_{7 i}$ & 0.3 & 0.3 & $100 \%$ & {$[\mathrm{FIX}]$} \\
\hline \multirow[t]{2}{*}{$5 s^{2} 8 g$} & $E_{\mathrm{av}}\left(5 s^{2} 8 g\right)$ & 483692 & 483686 & $100 \%$ & [FIX] \\
\hline & $\zeta_{8 g}$ & 0.3 & 0.3 & $100 \%$ & [FIX] \\
\hline \multirow[t]{2}{*}{$5 s^{2} 8 i$} & $E_{\mathrm{av}}\left(5 s^{2} 8 i\right)$ & 484460 & 483351 & $100 \%$ & \\
\hline & $\zeta_{8 i}$ & 0.2 & 0.2 & $100 \%$ & {$[\mathrm{FIX}]$} \\
\hline $5 s 5 p^{2}-5 s^{2} 5 d$ & $R^{1}(5 p, 5 p ; 5 s, 5 d)$ & 61452 & 43016 & $70 \%$ & {$[\mathrm{FIX}]$} \\
\hline $5 s 5 p^{2}-5 p^{2} 5 d$ & $R^{1}(5 s, 5 p ; 5 p, 5 d)$ & 62756 & 43929 & $70 \%$ & [FIX] \\
\hline $5 s 5 p^{2}-5 p^{2} 5 d$ & $R^{2}(5 s, 5 p ; 5 d, 5 p)$ & 45877 & 32114 & $70 \%$ & {$[\mathrm{FIX}]$} \\
\hline $5 s 5 p 4 f-5 s^{2} 6 d$ & $R^{3}(4 f, 5 p ; 5 s, 6 d)$ & -13554 & -10843 & $80 \%$ & [FIX] \\
\hline $5 s 5 p 4 f-5 s^{2} 6 d$ & $R^{1}(4 f, 5 p ; 6 d, 5 s)$ & -14268 & -11414 & $80 \%$ & {$[\mathrm{FIX}]$} \\
\hline
\end{tabular}

Notes.

${ }^{a}$ Parameters omitted from this table: direct, exchange, and CI integrals, and spin-orbit $\zeta$ parameters set to $100 \%$ of their HFR values. The HFR values for average energy parameters $E_{\mathrm{av}}$ were displaced by $19388 \mathrm{~cm}^{-1}$ in order to set the ground state at $0 \mathrm{~cm}^{-1}$. The standard deviation for the energy adjustment was $154 \mathrm{~cm}^{-1}$.

$\mathrm{b}$ The $5 p^{2} 5 d$ configuration does not have experimental levels.

sum (Equation (17.42) of TASS 1981) for the Rydberg series up to $4 d^{9}(n p+n f), n=30$, to obtain $\alpha=0.99 a_{0}^{3}$. For the Xe vII core, the values for polarizability prior to this work were the theoretical result $\alpha=4.52 a_{0}^{3}$ from Fraga \& Muszyńska's table and the experimental value $\alpha=4.2 \pm 0.8 a_{0}^{3}$ from Wang et al. (1996), who fitted the semiempirical polarizability formula from Edlén (1964) to the $5 s^{2} n l(n=5-8, l=4-7)$ energy levels. We tried a different approach, considering a more complete description to take into account levels with excited $4 d$ electrons, such as $[K r] 4 d^{9} 5 s^{2} n p$ and $4 d^{9} n f 5 s^{2}$, which have strong E1 transitions to the ground state. Using the same method as described above in this paragraph, this time with 8 even and 61 odd configurations including the Rydberg series for $[K r] 4 d^{10}(5 s n p+5 p n s)$ and $[K r] 4 d^{9} 5 s^{2}(n p+n f)$, we obtained $\alpha=5.80 a_{0}^{3}$. We used $r_{c}=0.86 a_{0}(4 d)$ for the Xe IX core and $r_{c}=1.69 a_{0}(5 s)$ for the Xe VII core.

\subsection{Transition Rates, Oscillator Strengths, and Lifetimes}

We calculated the weighted transition rates $(g A)$, the weighted oscillator strengths, and the cancellation factors using the HFR method with and without taking into account CP effects. When considering CP, we used $\alpha=0.99 a_{0}^{3}$ for transitions with the Xe IX core and $\alpha=5.80 a_{0}^{3}$ for transitions with the Xe viI core, as proposed above. Table 3 shows our results for the calculated values. The values for $g A$ differ by approximately $28 \%$ when the calculations with and without $\mathrm{CP}$ are compared. We compared our results for lifetimes with the experimental data from Biémont et al. (2005), as can be seen in Table 4. Among the 10 cases where experimental data are available, the results are better for calculations with $\mathrm{CP}$ in 8 cases. When we compared lifetimes obtained using $\alpha=4.52 a_{0}^{3}$, as considered by Biémont et al., and those obtained with $\alpha=5.80 a_{0}^{3}$ for the $\mathrm{Xe}$ VII core, both calculations showed similar results.

\section{ANALYSIS}

Table 3 shows the set of 243 lines observed in our experiments, 146 of which were determined for the first time. Another 37 lines, from Larsson et al. (1996), Wang et al. (1996), and Wang et al. (1997a), are shown with revised wavelengths as we measured them with a lower uncertainty. Observed line intensities given in this table are visual estimates of the blackening of the photographic plates. They do not take into account changes of sensitivity of our registration setup (including photographic plates and development process) with wavelength.

Prior to this work, a number of levels belonging to configurations $5 s 5 p^{2}$ and $5 p^{3}$, as well as $5 s^{2} n l$ up to $n=8$, were known. Configurations $5 s 5 p 5 d$ and $5 s 5 p 6 s$ have also been studied, but authors (Sarmiento et al. 1999, Reyna Almandos et al. (2001) on the one side, and Churilov \& Joshi 2000 on the other) disagreed on their conclusions about levels and lines.

Table 4 has a complete list of the 88 levels that were classified, described in the LS coupling scheme. In this paper, we revised 33 previously known energy levels. Levels with improved values were considered "revised," and those classified for the first time were considered "new" identifications. We also considered as "new" those levels that we reclassified from a set of lines completely different from the set of the previous classification, producing a significantly new energy value.

\subsection{Complex $n=5$}

The starting point for our analysis was the $5 s^{2} 5 p, 5 s 5 p^{2}$, and $5 s^{2} 5 d$ levels, as classified by Kaufman \& Sugar (1987), Tauheed et al. (1992), and Churilov \& Joshi (2000). This is a reliable set of levels determined from wavelengths measured with an uncertainty of only $0.005 \AA$. Other $n=5$ complex levels studied here belong to the $5 p^{3}, 5 s^{2}(5 f, 5 g)$, and $5 s 5 p 5 d$ configurations.

The level at $232585.5 \mathrm{~cm}^{-1}$, formerly classified as $5 p^{34} S_{3 / 2}$ by Sarmiento et al. (1999), has now been reclassified as ${ }^{2} D_{3 / 2}$. We also changed the levels $5 p^{32} D_{5 / 2}$ and ${ }^{2} P_{1 / 2}$, previously identified by only three lines (Churilov \& Joshi 2000), to the new values of $240517 \mathrm{~cm}^{-1}$ and $261029 \mathrm{~cm}^{-1}$, respectively, based on eight new lines.

The $5 s^{2} 5 f$ levels were first classified by Wang et al. (1996, 1997b). This identification was rejected by Churilov \& Joshi (2000) on the basis of three lines at $659.849,668.948$, and $671.357 \AA$ A. In agreement with Churilov \& Joshi (2000), Saloman (2004) reclassified another line, previously observed by Reyna Almandos et al. (2001) at $494.97 \AA$, as a $5 s 5 p^{2}-5 f$ transition. In the present work, we revise this level assignment based on six lines corresponding to transitions with the $5 d, 5 g$, and $6 g$ levels. It turned out that our level classification is in agreement with the original values by Wang et al. (1996, 1997b). 
Table 3

Transition Rates and Oscillator Strengths for Xe vI

\begin{tabular}{|c|c|c|c|c|c|c|c|c|c|c|c|c|}
\hline \multirow{2}{*}{$\begin{array}{l}\text { Line } \\
\text { Intens. }\end{array}$} & \multirow[t]{2}{*}{$\lambda(\AA)^{\mathrm{a}}$} & \multicolumn{2}{|c|}{ Designation } & \multicolumn{2}{|c|}{ Wavenumber $\left(\mathrm{cm}^{-1}\right)$} & \multicolumn{3}{|c|}{ HFR } & \multicolumn{3}{|c|}{$\mathrm{HFR}+\mathrm{CP}^{\mathrm{b}}$} & \multirow[t]{2}{*}{ Ref. $^{c}$} \\
\hline & & Lower Level & Upper Level & $\sigma_{\text {obs. }}$ & $\sigma_{\text {obs. }- \text { cal. }}$ & $\log (g f)$ & $g A\left(s^{-1}\right)$ & $\mathrm{CF}$ & $\log (g f)$ & $g A\left(s^{-1}\right)$ & $\mathrm{CF}$ & \\
\hline 1 & 418.84 & $5 s 5 p^{2}{ }^{2} D_{3 / 2}$ & $5 s 5 p 6 s\left({ }^{1} P\right){ }^{2} P_{1 / 2}$ & 238755 & 2 & -0.608 & $9.37(+09)$ & -0.266 & -0.529 & $1.12(+10)$ & -0.293 & New \\
\hline 2 & 422.28 & $5 s 5 p^{2}{ }^{4} P_{1 / 2}$ & $5 s 5 p 6 s\left({ }^{3} P\right)^{2} P_{1 / 2}$ & 236810 & -1 & -1.965 & $4.05(+08)$ & -0.068 & -1.985 & $3.87(+08)$ & -0.068 & New \\
\hline 1 & 427.96 & $5 s 5 p^{2}{ }^{4} P_{1 / 2}$ & $5 s 5 p 5 d\left({ }^{1} P\right){ }^{2} P_{3 / 2}$ & 233667 & 0 & -1.460 & $1.26(+09)$ & -0.037 & -1.350 & $1.62(+09)$ & -0.055 & New \\
\hline 4 & 436.58 & $5 s 5 p^{2}{ }^{4} P_{1 / 2}$ & $5 s 5 p 6 s\left({ }^{3} P\right){ }^{4} P_{3 / 2}$ & 229053 & 2 & -0.683 & $7.25(+09)$ & 0.205 & -0.611 & $8.54(+09)$ & 0.253 & New \\
\hline 1 & 445.38 & $5 s^{2} 5 d^{2} D_{3 / 2}$ & $5 s^{2} 6 f^{2} F_{5 / 2}$ & 224527 & 7 & -0.103 & $2.66(+10)$ & 0.517 & -0.316 & $1.63(+10)$ & 0.485 & New \\
\hline 12 & 447.473 & $5 s^{2} 5 p^{2} P_{1 / 2}$ & $5 s^{2} 6 s^{2} S_{1 / 2}$ & 223477 & -1 & -0.442 & $1.20(+10)$ & 0.869 & -0.234 & $1.94(+10)$ & 0.900 & (1) \\
\hline 1 & 458.77 & $5 s 5 p^{2}{ }^{4} P_{3 / 2}$ & $5 s 5 p 6 s\left({ }^{3} P\right){ }^{4} P_{1 / 2}$ & 217974 & 8 & -0.376 & $1.34(+10)$ & 0.507 & -0.297 & $1.60(+10)$ & 0.564 & New \\
\hline 2 & 461.76 & $5 s 5 p^{2}{ }^{4} P_{5 / 2}$ & $5 s 5 p 5 d\left({ }^{1} P\right){ }^{2} D_{5 / 2}$ & 216563 & -4 & -1.760 & $5.44(+08)$ & -0.014 & -1.856 & $4.36(+08)$ & -0.014 & New \\
\hline 1 & 465.12 & $5 s 5 p^{2}{ }^{2} D_{5 / 2}$ & $5 s 5 p 6 s\left({ }^{3} P\right){ }^{2} P_{3 / 2}$ & 214998 & -2 & 0.152 & $4.39(+10)$ & 0.585 & 0.203 & $4.93(+10)$ & 0.620 & New \\
\hline 5 & 466.34 & $5 s 5 p^{2}{ }^{4} P_{5 / 2}$ & $5 s 5 p 6 s\left({ }^{3} P\right){ }^{4} P_{3 / 2}$ & 214436 & 4 & -0.552 & $8.60(+09)$ & -0.268 & -0.482 & $1.01(+10)$ & -0.323 & New \\
\hline 1 & 469.37 & $5 s 5 p^{2}{ }^{4} P_{3 / 2}$ & $5 s 5 p 5 d\left({ }^{3} P\right){ }^{2} P_{1 / 2}$ & 213052 & 5 & -2.285 & $1.57(+08)$ & 0.024 & -2.097 & $2.42(+08)$ & 0.042 & $* \S(8)$ \\
\hline 9 & 481.054 & $5 s^{2} 5 p^{2} P_{3 / 2}$ & $5 s^{2} 6 s^{2} S_{1 / 2}$ & 207877 & -2 & -0.168 & $1.96(+10)$ & -0.883 & 0.038 & $3.14(+10)$ & -0.910 & (1) \\
\hline 1 & 482.34 & $5 s 5 p^{2}{ }^{2} S_{1 / 2}$ & $5 s 5 p 6 s\left({ }^{1} P\right){ }^{2} P_{3 / 2}$ & 207323 & -5 & -2.548 & $8.12(+07)$ & 0.006 & -2.447 & $1.02(+08)$ & 0.006 & New \\
\hline 2 & 496.58 & $5 s 5 p^{2}{ }^{2} D_{3 / 2}$ & $5 s 5 p 5 d\left({ }^{1} P\right){ }^{2} P_{3 / 2}$ & 201377 & -6 & -1.538 & $7.83(+08)$ & 0.011 & -1.689 & $5.53(+08)$ & 0.011 & New \\
\hline 4 & 502.76 & $5 s 5 p^{2}{ }^{2} D_{3 / 2}$ & $5 s 5 p 5 d\left({ }^{1} P\right){ }^{2} D_{5 / 2}$ & 198902 & 0 & -2.388 & $1.08(+08)$ & -0.001 & -2.359 & $1.15(+08)$ & -0.001 & $\S(8)$ \\
\hline 2 & 503.78 & $5 s 5 p^{2}{ }^{2} D_{3 / 2}$ & $5 s 5 p 5 d\left({ }^{1} P\right){ }^{2} D_{3 / 2}$ & 198499 & -1 & -2.282 & $1.35(+08)$ & -0.001 & -2.878 & $3.41(+07)$ & -0.001 & New \\
\hline 1 & 507.55 & $5 s 5 p^{2}{ }^{2} D_{5 / 2}$ & $5 s 5 p 5 d\left({ }^{1} P\right){ }^{2} P_{3 / 2}$ & 197025 & 2 & -2.848 & $3.68(+07)$ & 0.001 & -2.959 & $2.85(+07)$ & 0.001 & New \\
\hline 3 & 513.92 & $5 s 5 p^{2}{ }^{4} P_{1 / 2}$ & $5 s 5 p 5 d\left({ }^{3} P\right){ }^{4} P_{1 / 2}$ & 194583 & -1 & -2.344 & $1.14(+08)$ & -0.005 & -2.423 & $9.53(+07)$ & -0.005 & New \\
\hline 6 & 520.84 & $5 s 5 p^{2}{ }^{2} D_{3 / 2}$ & $5 s 5 p 5 d\left({ }^{1} P\right){ }^{2} F_{5 / 2}$ & 191998 & 0 & 0.589 & $9.54(+10)$ & -0.314 & 0.503 & $7.83(+10)$ & -0.337 & New \\
\hline 6 & 520.84 & $5 s^{2} 4 f^{2} F_{5 / 2}$ & $5 s^{2} 5 g^{2} G_{7 / 2}$ & 191998 & 1 & 0.489 & $7.59(+10)$ & 0.678 & 0.272 & $4.61(+10)$ & 0.651 & $*(4)$ \\
\hline 5 & 521.72 & $5 s^{2} 4 f^{2} F_{7 / 2}$ & $5 s^{2} 5 g^{2} G_{9 / 2}$ & 191674 & -4 & 0.599 & $9.72(+10)$ & 0.681 & 0.383 & $5.90(+10)$ & 0.653 & $*(4)$ \\
\hline 1 & 530.36 & $5 s 5 p^{2}{ }^{2} D_{3 / 2}$ & $5 s 5 p 5 d\left({ }^{3} P\right){ }^{2} P_{1 / 2}$ & 188551 & -4 & -0.972 & $2.53(+09)$ & -0.058 & -0.986 & $2.44(+09)$ & -0.067 & $\S(8)$ \\
\hline 2 & 533.18 & $5 s 5 p^{2}{ }^{2} P_{1 / 2}$ & $5 s 5 p 6 s\left({ }^{3} P\right){ }^{2} P_{1 / 2}$ & 187554 & -6 & -0.186 & $1.53(+10)$ & -0.504 & -0.186 & $1.53(+10)$ & -0.521 & New \\
\hline 1 & 533.56 & $5 s 5 p^{2}{ }^{4} P_{3 / 2}$ & $5 s 5 p 5 d\left({ }^{3} P\right){ }^{4} P_{3 / 2}$ & 187420 & -6 & -0.064 & $2.02(+10)$ & 0.523 & -0.166 & $1.60(+10)$ & 0.530 & New \\
\hline 2 & 536.74 & $5 s 5 p^{2}{ }^{4} P_{3 / 2}$ & $5 s 5 p 5 d\left({ }^{3} P\right){ }^{4} D_{5 / 2}$ & 186310 & 0 & 0.024 & $2.44(+10)$ & 0.167 & -0.082 & $1.91(+10)$ & 0.167 & New \\
\hline 6 & 537.612 & $5 s 5 p^{2}{ }^{2} D_{5 / 2}$ & $5 s 5 p 5 d\left({ }^{1} P\right){ }^{2} F_{7 / 2}$ & 186008 & -1 & 0.723 & $1.23(+11)$ & -0.341 & 0.638 & $1.01(+11)$ & -0.366 & (7) \\
\hline 6 & 543.510 & $5 s 5 p^{2}{ }^{4} P_{3 / 2}$ & $5 s 5 p 5 d\left({ }^{3} P\right)^{2} F_{5 / 2}$ & 183989 & 1 & -0.172 & $1.52(+10)$ & 0.368 & -0.279 & $1.19(+10)$ & 0.370 & (7) \\
\hline 2 & 544.55 & $5 s 5 p^{2}{ }^{4} P_{1 / 2}$ & $5 s 5 p 5 d\left({ }^{3} P\right){ }^{4} D_{1 / 2}$ & 183638 & -1 & 0.345 & $4.97(+10)$ & -0.868 & 0.239 & $3.89(+10)$ & -0.868 & New \\
\hline 1 & 545.35 & $5 s^{2} 5 d^{2} D_{3 / 2}$ & $5 s 5 p 6 s\left({ }^{1} P\right){ }^{2} P_{1 / 2}$ & 183368 & -5 & -1.951 & $2.51(+08)$ & 0.030 & -2.156 & $1.56(+08)$ & 0.017 & New \\
\hline 4 & 546.41 & $5 s^{2} 5 d^{2} D_{5 / 2}$ & $5 s 5 p 6 s\left({ }^{1} P\right){ }^{2} P_{3 / 2}$ & 183013 & -3 & -2.450 & $7.93(+07)$ & 0.007 & -2.961 & $2.44(+07)$ & 0.002 & New \\
\hline 5 & 547.78 & $5 s 5 p^{2}{ }^{4} P_{1 / 2}$ & $5 s 5 p 5 d\left({ }^{3} P\right){ }^{4} D_{3 / 2}$ & 182555 & -4 & 0.467 & $6.53(+10)$ & 0.791 & 0.362 & $5.12(+10)$ & 0.794 & New \\
\hline 3 & 550.86 & $5 s 5 p^{2}{ }^{2} P_{1 / 2}$ & $5 s 5 p 5 d\left({ }^{1} P\right){ }^{2} D_{3 / 2}$ & 181534 & 1 & -0.374 & $9.12(+09)$ & 0.079 & -0.474 & $7.23(+09)$ & 0.077 & New \\
\hline 1 & 553.70 & $5 s 5 p^{2}{ }^{4} P_{5 / 2}$ & $5 s 5 p 5 d\left({ }^{3} P\right){ }^{4} P_{3 / 2}$ & 180603 & 4 & -0.149 & $1.55(+10)$ & -0.485 & -0.250 & $1.22(+10)$ & -0.488 & New \\
\hline 12 & 554.785 & $5 s^{2} 5 p^{2} P_{1 / 2}$ & $5 s^{2} 5 d^{2} D_{3 / 2}$ & 180 & 0 & & & -0.6 & 0.041 & $2.38(+10)$ & -0.553 & (1) \\
\hline 1 & 557.15 & $5 s 5 p^{2}{ }^{4} P_{5 / 2}$ & $5 s 5 p 5 d\left({ }^{3} P\right){ }^{4} D_{5 / 2}$ & 179485 & 2 & 0.579 & $8.17(+10)$ & -0.829 & 0.474 & $6.41(+10)$ & -0.830 & New \\
\hline 1 & 561.33 & $5 s 5 p^{2}{ }^{4} P_{5 / 2}$ & $5 s 5 p 5 d\left({ }^{3} P\right){ }^{4} D_{7 / 2}$ & 178148 & -2 & 0.899 & $1.68(+11)$ & 0.851 & 0.794 & $1.32(+11)$ & 0.854 & New \\
\hline 2 & 568.69 & $5 s 5 p^{2}{ }^{4} P_{3 / 2}$ & $5 s 5 p 5 d\left({ }^{3} P\right){ }^{4} D_{1 / 2}$ & 175843 & -4 & -1.188 & $1.33(+09)$ & 0.069 & -1.295 & $1.04(+09)$ & 0.069 & New \\
\hline 3 & 572.20 & $5 s 5 p^{2}{ }^{4} P_{3 / 2}$ & $5 s 5 p 5 d\left({ }^{3} P\right){ }^{4} D_{3 / 2}$ & 174764 & -3 & -0.128 & $1.52(+10)$ & -0.236 & -0.235 & $1.19(+10)$ & -0.236 & New \\
\hline 5 & 574.23 & $5 s 5 p^{2}{ }^{4} P_{1 / 2}$ & $5 p^{3}{ }^{2} P_{3 / 2}$ & 174146 & -6 & -1.094 & $1.63(+09)$ & -0.093 & -1.220 & $1.22(+09)$ & -0.094 & $\dagger(8)$ \\
\hline 8 & 577.252 & $5 s 5 p^{2}{ }^{4} P_{3 / 2}$ & $5 s 5 p 5 d\left({ }^{3} P\right){ }^{4} P_{5 / 2}$ & 173235 & -1 & 0.605 & $8.06(+10)$ & 0.832 & 0.499 & $6.32(+10)$ & 0.833 & (7) \\
\hline 2 & 582.80 & $5 s 5 p^{2}{ }^{2} P_{1 / 2}$ & $5 s 5 p 5 d\left({ }^{3} P\right){ }^{2} P_{1 / 2}$ & 171585 & -3 & -1.029 & $1.84(+09)$ & -0.029 & -1.180 & $1.30(+09)$ & -0.026 & $\S(8)$ \\
\hline 3 & 584.47 & $5 s 5 p^{2}{ }^{2} D_{5 / 2}$ & $5 s 5 p 5 d\left({ }^{3} P\right){ }^{2} F_{7 / 2}$ & 171095 & -3 & 0.506 & $6.28(+10)$ & 0.213 & 0.401 & $4.93(+10)$ & 0.217 & New \\
\hline 3 & 593.69 & $5 s 5 p^{2}{ }^{4} P_{1 / 2}$ & $5 p^{32} P_{1 / 2}$ & 168438 & -5 & -2.897 & $2.39(+07)$ & -0.007 & -3.120 & $1.43(+07)$ & -0.006 & New \\
\hline 5 & 598.211 & $5 s 5 p^{2}{ }^{2} D_{3 / 2}$ & $5 s 5 p 5 d\left({ }^{3} P\right)^{2} D_{5 / 2}$ & 167165 & -4 & -0.977 & $1.96(+09)$ & -0.014 & -1.045 & $1.68(+09)$ & -0.015 & (7) \\
\hline 12 & 599.848 & $5 s^{2} 5 p^{2} P_{3 / 2}$ & $5 s^{2} 5 d^{2} D_{5 / 2}$ & 166709 & 0 & 0.734 & $1.01(+11)$ & -0.749 & 0.299 & $3.69(+10)$ & -0.680 & (1) \\
\hline 1 & 603.74 & $5 s 5 p^{2}{ }^{2} P_{1 / 2}$ & $5 s 5 p 5 d\left({ }^{3} P\right){ }^{2} P_{3 / 2}$ & & -5 & & & 0.7 & 0.491 & $5.66(+10)$ & 0.735 & New \\
\hline 7 & 606.310 & $5 s 5 p^{2}{ }^{2} P_{3 / 2}$ & $5 s 5 p 5 d\left({ }^{1} P\right){ }^{2} P_{1 / 2}$ & 164932 & -1 & -0.166 & $1.24(+10)$ & -0.392 & -0.293 & $9.23(+09)$ & -0.387 & $\S(7)$ \\
\hline 8 & 607.348 & $5 s^{2} 5 p^{2} P_{3 / 2}$ & $5 s^{2} 5 d^{2} D_{3 / 2}$ & 164650 & -1 & -0.065 & $1.56(+10)$ & -0.713 & -0.443 & $6.52(+09)$ & -0.654 & (1) \\
\hline 2 & 613.13 & $5 s 5 p^{2}{ }^{4} P_{5 / 2}$ & $5 s^{2} 6 p^{2} P_{3 / 2}$ & 163098 & -3 & -1.821 & $2.68(+08)$ & -0.023 & -1.699 & $3.55(+08)$ & -0.038 & New \\
\hline 4 & 613.75 & $5 s 5 p^{2}{ }^{2} D_{3 / 2}$ & $5 s 5 p 5 d\left({ }^{3} P\right){ }^{4} P_{3 / 2}$ & 162933 & -1 & 0.070 & $2.08(+10)$ & 0.557 & -0.052 & $1.56(+10)$ & 0.557 & New \\
\hline 6 & 614.222 & $5 s 5 p^{2}{ }^{2} D_{5 / 2}$ & $5 s 5 p 5 d\left({ }^{3} P\right)^{2} D_{5 / 2}$ & 162808 & -1 & 0.601 & $7.06(+10)$ & 0.671 & 0.482 & $5.37(+10)$ & 0.671 & (7) \\
\hline 3 & 615.30 & $5 s 5 p^{2}{ }^{2} P_{3 / 2}$ & $5 s 5 p 6 s\left({ }^{3} P\right){ }^{4} P_{3 / 2}$ & 162522 & -3 & -1.133 & $1.29(+09)$ & -0.016 & -1.231 & $1.03(+09)$ & -0.016 & New \\
\hline 2 & 617.59 & $5 s^{2} 5 d^{2} D_{5 / 2}$ & $5 s 5 p 6 s\left({ }^{3} P\right){ }^{2} P_{3 / 2}$ & 161920 & -2 & -0.468 & $5.96(+09)$ & -0.456 & -0.506 & $5.46(+09)$ & -0.437 & New \\
\hline 4 & 626.970 & $5 s 5 p^{2}{ }^{2} D_{3 / 2}$ & $5 s 5 p 5 d\left({ }^{3} P\right){ }^{2} F_{5 / 2}$ & 159497 & 1 & 0.381 & $4.08(+10)$ & 0.253 & 0.268 & $3.15(+10)$ & 0.255 & (7) \\
\hline 10 & 628.489 & $5 s^{2} 5 p^{2} P_{1 / 2}$ & $5 s 5 p^{2}{ }^{2} P_{3 / 2}$ & 159112 & 0 & -0.138 & $1.23(+10)$ & 0.292 & -0.364 & $7.32(+09)$ & 0.267 & (1) \\
\hline 4 & 628.91 & $5 s 5 p^{2}{ }^{4} P_{3 / 2}$ & $5 s 5 p 5 d\left({ }^{3} P\right){ }^{4} F_{3 / 2}$ & 159005 & -1 & -1.148 & $1.20(+09)$ & -0.626 & -1.266 & $9.13(+08)$ & -0.627 & New \\
\hline 8 & 632.930 & $5 s^{2} 5 p^{2} P_{1 / 2}$ & $5 s 5 p^{2} S_{1 / 2}$ & 157995 & -1 & -1.080 & $1.39(+09)$ & 0.034 & -1.219 & $1.01(+09)$ & 0.034 & (1) \\
\hline 3 & 637.67 & $5 s 5 p^{2} D_{5 / 2}$ & $5 s 5 p 5 d\left({ }^{3} P\right)^{2} D_{3 / 2}$ & 156821 & 1 & -1.937 & $1.91(+08)$ & -0.013 & -2.094 & $1.33(+08)$ & -0.012 & $\S(8)$ \\
\hline 2 & 640.50 & $5 s 5 p^{2}{ }^{2} D_{5 / 2}$ & $5 s 5 p 5 d\left({ }^{3} P\right){ }^{4} D_{7 / 2}$ & 156128 & 3 & -0.340 & $7.43(+09)$ & 0.219 & -0.455 & $5.71(+09)$ & 0.217 & New \\
\hline 8 & 643.36 & $5 s 5 p^{2}{ }^{2} S_{1 / 2}$ & $5 s 5 p 5 d\left({ }^{3} P\right){ }^{2} P_{1 / 2}$ & 155434 & 5 & 0.243 & $2.82(+10)$ & -0.526 & 0.127 & $2.15(+10)$ & -0.510 & New \\
\hline 7 & 643.748 & $5 s 5 p^{2}{ }^{4} P_{5 / 2}$ & $5 s 5 p 5 d\left({ }^{3} P\right){ }^{4} F_{5 / 2}$ & 155340 & -1 & -0.871 & $2.16(+09)$ & -0.584 & -0.985 & $1.67(+09)$ & -0.583 & (7) \\
\hline 6 & 644.59 & $5 s 5 p^{2}{ }^{2} D_{5 / 2}$ & $5 s 5 p 5 d\left({ }^{3} P\right){ }^{2} F_{5 / 2}$ & 155137 & 1 & -0.295 & $8.18(+09)$ & 0.117 & -0.435 & $5.92(+09)$ & 0.112 & New \\
\hline 4 & 648.03 & $5 s 5 p^{2}{ }^{2} P_{3 / 2}$ & $5 s 5 p 5 d\left({ }^{3} P\right){ }^{2} P_{1 / 2}$ & 154314 & 1 & -0.974 & $1.68(+09)$ & -0.111 & -1.076 & $1.33(+09)$ & -0.110 & $\S(8)$ \\
\hline 2 & 657.10 & $5 s 5 p^{2}{ }^{4} P_{5 / 2}$ & $5 s 5 p 5 d\left({ }^{3} P\right){ }^{4} F_{3 / 2}$ & 152184 & 5 & -2.493 & $4.97(+07)$ & 0.057 & -2.583 & $4.05(+07)$ & 0.063 & New \\
\hline 6 & 659.22 & $5 s^{2} 5 d^{2} D_{3 / 2}$ & $5 s^{2} 5 f^{2} F_{5 / 2}$ & 151694 & -1 & 0.881 & $1.17(+11)$ & 0.680 & 0.767 & $8.97(+10)$ & 0.690 & $*(5)$ \\
\hline
\end{tabular}


Table 3

(Continued)

\begin{tabular}{|c|c|c|c|c|c|c|c|c|c|c|c|c|}
\hline \multirow{2}{*}{$\begin{array}{l}\text { Line } \\
\text { Intens. }\end{array}$} & \multirow[t]{2}{*}{$\lambda(\AA)^{\mathrm{a}}$} & \multicolumn{2}{|c|}{ Designation } & \multicolumn{2}{|c|}{ Wavenumber $\left(\mathrm{cm}^{-1}\right)$} & \multicolumn{3}{|c|}{ HFR } & \multicolumn{3}{|c|}{$\mathrm{HFR}+\mathrm{CP}^{\mathrm{b}}$} & \multirow[t]{2}{*}{ Ref. } \\
\hline & & Lower Level & Upper Level & $\sigma_{\text {obs. }}$ & $\sigma_{\text {obs. }- \text { cal. }}$ & $\log (g f)$ & $g A\left(s^{-1}\right)$ & $\mathrm{CF}$ & $\log (g f)$ & $g A\left(s^{-1}\right)$ & $\mathrm{CF}$ & \\
\hline 4 & 668.99 & $5 s 5 p^{2}{ }^{2} S_{1 / 2}$ & $5 s 5 p 5 d\left({ }^{3} P\right){ }^{2} P_{3 / 2}$ & 149479 & -1 & -0.686 & $3.06(+09)$ & 0.040 & -0.820 & $2.25(+09)$ & 0.038 & New \\
\hline 4 & 669.64 & $5 s^{2} 5 d^{2} D_{5 / 2}$ & $5 s^{2} 5 f^{2} F_{7 / 2}$ & 149334 & 0 & 1.012 & $1.53(+11)$ & 0.726 & 0.899 & $1.18(+11)$ & 0.735 & $*(5)$ \\
\hline 6 & 670.47 & $5 s^{2} 5 d^{2} D_{3 / 2}$ & $5 s 5 p 6 s\left({ }^{3} P\right){ }^{2} P_{1 / 2}$ & 149149 & 2 & -0.782 & $2.45(+09)$ & -0.315 & -0.849 & $2.10(+09)$ & -0.294 & New \\
\hline 4 & 672.32 & $5 s 5 p^{2}{ }^{2} D_{3 / 2}$ & $5 s 5 p 5 d\left({ }^{3} P\right){ }^{4} P_{5 / 2}$ & 148739 & -5 & -1.397 & $5.91(+08)$ & -0.049 & -1.538 & $4.27(+08)$ & -0.047 & New \\
\hline 3 & 674.02 & $5 s 5 p^{2}{ }^{2} P_{3 / 2}$ & $5 s 5 p 5 d\left({ }^{3} P\right){ }^{2} P_{3 / 2}$ & 148364 & 0 & -0.070 & $1.25(+10)$ & 0.218 & -0.201 & $9.22(+09)$ & 0.212 & New \\
\hline 6 & 677.720 & $5 s 5 p^{2}{ }^{4} P_{1 / 2}$ & $5 p^{3}{ }^{4} S_{3 / 2}$ & 147554 & 0 & -0.390 & $5.90(+09)$ & 0.517 & -0.551 & $4.07(+09)$ & 0.507 & (7) \\
\hline 3 & 684.88 & $5 s^{2} 5 d^{2} D_{3 / 2}$ & $5 s 5 p 5 d\left({ }^{1} P\right){ }^{2} P_{3 / 2}$ & 146011 & 8 & -0.063 & $1.23(+10)$ & 0.419 & -0.242 & $8.15(+09)$ & 0.385 & New \\
\hline 3 & 87.59 & $5 s 5 p^{2}{ }^{2} D_{3 / 2}$ & $5 s^{2} 6 p^{2} P_{3 / 2}$ & 145436 & 0 & -1.457 & $4.91(+08)$ & 0.035 & -1.456 & $4.93(+08)$ & 0.045 & $\dagger(8)$ \\
\hline 2 & 688.11 & $5 s 5 p^{2}{ }^{2} P_{1 / 2}$ & $5 s 5 p 5 d\left({ }^{3} P\right){ }^{4} P_{1 / 2}$ & 145326 & -7 & -4.545 & $4.03(+05)$ & 0.001 & -4.619 & $3.40(+05)$ & 0.001 & New \\
\hline 5 & 692.591 & $5 s 5 p^{2}{ }^{2} D_{5 / 2}$ & $5 s 5 p 5 d\left({ }^{3} P\right){ }^{4} P_{5 / 2}$ & 144385 & 1 & -0.438 & $5.08(+09)$ & -0.134 & -0.574 & $3.72(+09)$ & -0.128 & (7) \\
\hline 3 & 693.42 & $5 s 5 p^{2}{ }^{2} P_{1 / 2}$ & $5 s 5 p 5 d\left({ }^{3} P\right){ }^{2} D_{3 / 2}$ & 144213 & 0 & -0.382 & $5.79(+09)$ & 0.135 & -0.507 & $4.35(+09)$ & 0.133 & New \\
\hline 3 & 695.42 & $5 s^{2} 5 d^{2} D_{3 / 2}$ & $5 s 5 p 5 d\left({ }^{1} P\right){ }^{2} P_{1 / 2}$ & 143798 & 3 & 0.024 & $1.46(+10)$ & 0.269 & -0.196 & $8.78(+09)$ & 0.225 & New \\
\hline 14 & 696.801 & $5 s^{2} 5 p^{2} P_{3 / 2}$ & $5 s 5 p^{2}{ }^{2} P_{3 / 2}$ & 143513 & 0 & 0.534 & $4.71(+10)$ & -0.510 & 0.394 & $3.41(+10)$ & -0.534 & (1) \\
\hline 4 & 698.69 & $5 s^{2} 5 d^{2} D_{3 / 2}$ & $5 s 5 p 5 d\left({ }^{1} P\right){ }^{2} D_{3 / 2}$ & 143125 & 5 & -0.313 & $6.48(+09)$ & -0.158 & -0.445 & $4.78(+09)$ & -0.158 & $\S(8)$ \\
\hline 12 & 702.264 & $5 s^{2} 5 p^{2} P_{3 / 2}$ & $5 s 5 p^{2}{ }^{2} S_{1 / 2}$ & 142397 & 0 & 0.090 & $1.67(+10)$ & 0.551 & -0.069 & $1.16(+10)$ & 0.535 & (1) \\
\hline 5 & 704.90 & $5 s 5 p^{2}{ }^{2} D_{3 / 2}$ & $5 p^{3}{ }^{2} P_{3 / 2}$ & 141864 & -4 & -1.115 & $1.03(+09)$ & -0.028 & -1.359 & $5.87(+08)$ & -0.021 & New \\
\hline 12 & 705.035 & $5 s^{2} 5 p^{2} P_{1 / 2}$ & $5 s 5 p^{2}{ }^{2} P_{1 / 2}$ & 141837 & 0 & 0.205 & $2.15(+10)$ & -0.595 & 0.049 & $1.50(+10)$ & -0.587 & (1) \\
\hline 3 & 706.87 & $5 s^{2} 5 d^{2} D_{5 / 2}$ & $5 s 5 p 5 d\left({ }^{1} P\right){ }^{2} D_{5 / 2}$ & 141469 & 5 & 0.435 & $3.63(+10)$ & -0.381 & 0.266 & $2.46(+10)$ & -0.359 & $\S(8)$ \\
\hline 5 & 708.842 & $5 s 5 p^{2}{ }^{2} D_{5 / 2}$ & $5 s^{2} 6 p^{2} P_{3 / 2}$ & 141075 & -1 & -0.137 & $9.70(+09)$ & 0.334 & & & 0.368 & (7) \\
\hline 3 & 713.55 & $5 s 5 p^{2}{ }^{4} P_{3 / 2}$ & $5 p^{3} 2 D_{5 / 2}$ & 140144 & 5 & -2.3 & $5.79(+07)$ & -0. & -2 & $4.13(+07)$ & -0.060 & New \\
\hline 3 & 713.55 & $5 s^{2} 6 s^{2} S_{1 / 2}$ & $5 s 5 p 6 s\left({ }^{1} P\right){ }^{2} P_{1 / 2}$ & & -1 & 0.013 & $1.35(+$ & -0 & -0.078 & $1.09(+10)$ & -0.513 & New \\
\hline 6 & 714.172 & $5 s 5 p^{2}{ }^{2} D_{3 / 2}$ & $5 s^{2} 6 p^{2} P_{1 / 2}$ & 140022 & 1 & -0.422 & $4.94(+09)$ & 0.360 & -0.458 & $4.55(+09)$ & 0.394 & (7) \\
\hline 6 & 714.289 & $5 s 5 p^{2}{ }^{4} P_{1 / 2}$ & $5 p^{3}{ }^{2} D_{3 / 2}$ & 139999 & -1 & -0.436 & $4.79(+09)$ & -0.479 & -0.604 & $3.25(+09)$ & -0.458 & (7) \\
\hline 8 & 715.507 & $5 s 5 p^{2}{ }^{4} P_{3 / 2}$ & $5 p^{3}{ }^{4} S_{3 / 2}$ & 139761 & -1 & -0.003 & $1.29(+10)$ & 0.657 & -0.169 & $8.81(+09)$ & 0.638 & (7) \\
\hline 5 & 17.75 & $5 s^{2} 5 d^{2} D_{5 / 2}$ & $5 s 5 p 6 s\left({ }^{3} P\right){ }^{4} P_{3 / 2}$ & 139324 & -5 & -0.341 & $5.89(+09)$ & 0.174 & -0.615 & $3.13(+09)$ & 0.122 & New \\
\hline 2 & 18.03 & $5 s^{2} 6 p^{2} P_{1 / 2}$ & $5 s^{2} 7 d^{2} D_{3 / 2}$ & 139270 & 2 & -1.411 & $5.01(+08)$ & 0.030 & -1.166 & $8.81(+08)$ & 0.087 & New \\
\hline 2 & 724.14 & $5 s^{2} 5 d^{2} D_{3 / 2}$ & $5 s 5 p 6 s\left({ }^{3} P\right){ }^{4} P_{1 / 2}$ & 138095 & 1 & -3.343 & $5.79(+06)$ & 0.008 & -3.068 & $1.09(+07)$ & 0.017 & New \\
\hline 1 & 725.25 & $5 s 5 p 5 d\left({ }^{3} P\right){ }^{4} F_{7 / 2}$ & $5 s^{2} 7 d^{2} D_{5 / 2}$ & 137883 & 1 & -2.697 & $2.55(+07)$ & 0.022 & -2.988 & $1.31(+07)$ & 0.011 & New \\
\hline 2 & 726.12 & $5 s 5 p^{2}{ }^{2} D_{5 / 2}$ & $5 s 5 p 5 d\left({ }^{3} P\right){ }^{4} F_{7 / 2}$ & 137718 & 0 & -3.681 & $2.65(+06)$ & 0.000 & -4.492 & $4.09(+05)$ & 0.000 & New \\
\hline 4 & 727.237 & $5 s 5 p^{2}{ }^{2} D_{5 / 2}$ & $5 p^{32} P_{3 / 2}$ & 137507 & -1 & -0.269 & $6.81(+09)$ & -0.131 & -0.489 & $4.11(+09)$ & -0.104 & (7) \\
\hline 5 & 731.96 & $5 s^{2} 5 d^{2} D_{3 / 2}$ & $5 s 5 p 5 d\left({ }^{1} P\right)^{2} F_{5 / 2}$ & 136619 & 1 & 0.028 & $1.33(+10)$ & 0.086 & -0.193 & $8.01(+09)$ & 0.071 & New \\
\hline 4 & 734.48 & $5 s 5 p^{2}{ }^{2} D_{3 / 2}$ & $5 p^{32} P_{1 / 2}$ & & -8 & -0.2 & $6.90(+09)$ & -0.217 & -0.448 & $4.39(+09)$ & -0.185 & New \\
\hline 4 & 743.43 & $5 s 5 p^{2}{ }^{2} D_{3 / 2}$ & $5 s 5 p 5 d\left({ }^{3} P\right){ }^{4} F_{3 / 2}$ & 4512 & -2 & -1.128 & $8.98(+08)$ & 0.293 & -1.253 & $6.72(+08)$ & 0.294 & New \\
\hline 6 & 750.13 & $5 s 5 p^{2}{ }^{2} P_{1 / 2}$ & $5 s 5 p 5 d\left({ }^{3} P\right){ }^{4} D_{3 / 2}$ & 133310 & 2 & -1.747 & $2.13(+08)$ & -0.017 & -1.846 & $1.70(+08)$ & -0.017 & New \\
\hline 6 & 750.13 & $5 s 5 p^{2}{ }^{4} P_{5 / 2}$ & $5 p^{3}{ }^{2} D_{5 / 2}$ & 133310 & -2 & -0.937 & $1.37(+09)$ & 0.145 & -1.136 & $8.69(+08)$ & 0.127 & New \\
\hline 6 & 50.13 & $5 s 5 p^{2}{ }^{2} D_{5 / 2}$ & $5 s 5 p 5 d\left({ }^{3} P\right){ }^{4} F_{5 / 2}$ & 133310 & -6 & -1.222 & $7.10(+08)$ & 0.221 & -1.357 & $5.21(+08)$ & 0.216 & New \\
\hline 4 & 750.92 & $5 s^{2} 5 d^{2} D_{3 / 2}$ & $5 s 5 p 5 d\left({ }^{3} P\right){ }^{2} P_{1 / 2}$ & 133172 & -3 & -1.789 & $1.92(+08)$ & -0.020 & -2.463 & $4.07(+07)$ & -0.005 & $* \S(8)$ \\
\hline 8 & 52.247 & $5 s 5 p^{2}{ }^{4} P_{5 / 2}$ & $5 p^{3}{ }^{4} S_{3 / 2}$ & 132935 & 0 & -0.001 & $1.18(+10)$ & 0.531 & -0.162 & $8.12(+09)$ & 0.522 & (7) \\
\hline 6 & 756.391 & $5 s 5 p^{2}{ }^{4} P_{3 / 2}$ & $5 p^{3}{ }^{2} D_{3 / 2}$ & 132207 & -1 & -0.367 & $5.01(+09)$ & -0.410 & -0.528 & $3.46(+09)$ & -0.402 & (7) \\
\hline 4 & 770.36 & $5 s 5 p^{2}{ }^{2} S_{1 / 2}$ & $5 s 5 p 5 d\left({ }^{3} P\right){ }^{4} P_{3 / 2}$ & 129809 & 1 & -1.416 & $4.29(+08)$ & -0.029 & -1.552 & $3.14(+08)$ & -0.028 & New \\
\hline 5 & 774.13 & $5 s 5 p^{2}{ }^{2} S_{1 / 2}$ & $5 s 5 p 5 d\left({ }^{3} P\right){ }^{4} P_{1 / 2}$ & 129177 & 3 & -3.778 & $1.86(+06)$ & -0.009 & -3.894 & $1.42(+06)$ & -0.009 & New \\
\hline 7 & 778.44 & $5 s 5 p^{2}{ }^{2} P_{1 / 2}$ & $5 s^{2} 6 p^{2} P_{3 / 2}$ & 128462 & -7 & -1.117 & $8.42(+08)$ & -0.073 & -1.285 & $5.72(+08)$ & -0.065 & $*(4)$ \\
\hline 5 & 780.89 & $5 s 5 p^{2}{ }^{2} S_{1 / 2}$ & $5 s 5 p 5 d\left({ }^{3} P\right){ }^{2} D_{3 / 2}$ & 128059 & 5 & -1.139 & $7.98(+08)$ & -0.041 & -1.276 & $5.82(+08)$ & -0.039 & New \\
\hline 5 & 780.89 & $5 s 5 p^{2}{ }^{2} P_{3 / 2}$ & $5 s 5 p 5 d\left({ }^{3} P\right){ }^{4} P_{1 / 2}$ & 1280 & 1 & -2.2 & $5.89(+07)$ & 0.546 & -2.372 & $4.64(+07)$ & 0.539 & New \\
\hline 3 & 783.87 & $5 s 5 p^{2}{ }^{2} P_{3 / 2}$ & $5 s 5 p 5 d\left({ }^{3} P\right){ }^{4} D_{5 / 2}$ & 127572 & -4 & -1.921 & $1.30(+08)$ & -0.032 & -2.034 & $1.00(+08)$ & -0.033 & New \\
\hline 3 & 783.91 & $5 s 5 p 5 d\left({ }^{3} P\right){ }^{2} D_{3 / 2}$ & $5 s^{2} 8 s^{2} S_{1 / 2}$ & 127566 & 3 & -2.671 & $2.30(+07)$ & -0.125 & -2.707 & $2.12(+07)$ & -0.124 & New \\
\hline 5 & 785.99 & $5 s^{2} 5 d^{2} D_{3 / 2}$ & $5 s 5 p 5 d\left({ }^{3} P\right){ }^{2} P_{3 / 2}$ & 127228 & 2 & -0.641 & $2.46(+09)$ & -0.126 & -0.847 & $1.53(+09)$ & -0.107 & New \\
\hline 8 & 792.149 & $5 s^{2} 5 p^{2} P_{3 / 2}$ & $5 s 5 p^{2}{ }^{2} P_{1 / 2}$ & 126239 & 1 & -1.332 & $4.94(+08)$ & -0.024 & -1.469 & $3.60(+08)$ & -0.025 & (1) \\
\hline 1 & 794.86 & $5 s 5 p 5 d\left({ }^{3} P\right){ }^{4} P_{3 / 2}$ & $5 s^{2} 8 s^{2} S_{1 / 2}$ & 125808 & -1 & -3.033 & $9.80(+06)$ & -0.078 & -3.062 & $9.17(+06)$ & -0.079 & New \\
\hline 5 & 795.28 & $5 s 5 p 5 d\left({ }^{1} P\right){ }^{2} F_{5 / 2}$ & $5 s^{2} 8 d^{2} D_{3 / 2}$ & 125742 & -3 & -1.624 & $2.50(+08)$ & 0.017 & -2.134 & $7.72(+07)$ & 0.006 & New \\
\hline 6 & 797.571 & $5 s 5 p^{2}{ }^{4} P_{5 / 2}$ & $5 p^{3}{ }^{2} D_{3 / 2}$ & 125381 & 0 & 0.008 & $1.07(+10)$ & -0.601 & -0.156 & $7.34(+09)$ & -0.584 & (7) \\
\hline 5 & 798.37 & $5 s 5 p^{2}{ }^{2} P_{3 / 2}$ & $5 s 5 p 5 d\left({ }^{3} P\right){ }^{2} F_{5 / 2}$ & 125255 & 1 & -0.853 & $1.47(+09)$ & 0.037 & -0.990 & $1.07(+09)$ & 0.036 & New \\
\hline 10 & 800.65 & $5 s 5 p^{2}{ }^{2} P_{1 / 2}$ & $5 p^{3}{ }^{2} P_{3 / 2}$ & 124899 & -2 & -1.008 & $1.03(+09)$ & 0.025 & -1.202 & $6.57(+08)$ & 0.021 & New \\
\hline 5 & 800.832 & $5 s^{2} 5 p^{2} P_{1 / 2}$ & $5 s 5 p^{2}{ }^{2} D_{3 / 2}$ & 124870 & 0 & -0.177 & $6.94(+09)$ & 0.208 & -0.203 & $6.54(+09)$ & 0.313 & (1) \\
\hline 6 & 812.67 & $5 s 5 p^{2}{ }^{2} P_{1 / 2}$ & $5 s^{2} 6 p^{2} P_{1 / 2}$ & 123051 & -3 & -1.930 & $1.19(+08)$ & -0.025 & -2.138 & $7.37(+07)$ & -0.020 & New \\
\hline 3 & 839.00 & $5 s 5 p^{2}{ }^{2} P_{1 / 2}$ & $5 p^{32} P_{1 / 2}$ & 119190 & -2 & -1.380 & $3.96(+08)$ & 0.015 & -1.564 & $2.59(+08)$ & 0.014 & New \\
\hline 2 & 845.85 & $5 s 5 p^{2}{ }^{2} S_{1 / 2}$ & $5 s 5 p 5 d\left({ }^{3} P\right){ }^{4} D_{1 / 2}$ & 118224 & -5 & -5.244 & $5.29(+04)$ & 0.000 & -6.034 & $8.59(+03)$ & 0.000 & New \\
\hline 2 & 846.67 & $5 s 5 p 5 d\left({ }^{3} P\right){ }^{2} D_{3 / 2}$ & $5 s^{2} 7 d^{2} D_{3 / 2}$ & 118110 & 1 & -2.892 & $1.19(+07)$ & -0.017 & -2.887 & $1.20(+07)$ & -0.020 & New \\
\hline 5 & 847.38 & $5 s^{2} 5 d^{2} D_{5 / 2}$ & $5 s 5 p 5 d\left({ }^{3} P\right){ }^{2} F_{7 / 2}$ & 118011 & -3 & -0.698 & $1.86(+09)$ & 0.037 & -0.960 & $1.02(+09)$ & 0.028 & New \\
\hline 3 & 853.59 & $5 s 5 p^{2}{ }^{2} S_{1 / 2}$ & $5 s 5 p 5 d\left({ }^{3} P\right){ }^{4} D_{3 / 2}$ & 117152 & 3 & -2.357 & $4.03(+07)$ & 0.007 & -2.549 & $2.59(+07)$ & 0.005 & New \\
\hline 1 & 853.90 & $5 s 5 p^{2}{ }^{2} P_{3 / 2}$ & $5 s 5 p 5 d\left({ }^{3} P\right){ }^{4} D_{1 / 2}$ & 117110 & -3 & -3.179 & $6.04(+06)$ & 0.010 & -3.328 & $4.28(+06)$ & 0.009 & New \\
\hline 4 & 873.38 & $5 s 5 p^{2}{ }^{2} P_{3 / 2}$ & $5 s 5 p 5 d\left({ }^{3} P\right){ }^{4} P_{5 / 2}$ & 114498 & -4 & -1.546 & $2.48(+08)$ & -0.017 & -1.706 & $1.72(+08)$ & -0.016 & New \\
\hline 11 & 880.043 & $5 s^{2} 5 p^{2} P_{3 / 2}$ & $5 s 5 p^{2}{ }^{2} D_{5 / 2}$ & 113631 & 0 & -0.215 & $5.24(+09)$ & 0.158 & -0.205 & $5.36(+09)$ & 0.264 & (1) \\
\hline 8 & 886.63 & $5 s 5 p 5 d\left({ }^{3} P\right){ }^{2} D_{5 / 2}$ & $5 s^{2} 7 d^{2} D_{5 / 2}$ & 112787 & -4 & -3.942 & $9.73(+05)$ & 0.001 & -3.918 & $1.03(+06)$ & 0.001 & New \\
\hline 1 & 890.38 & $5 s 5 p^{2}{ }^{2} S_{1 / 2}$ & $5 s^{2} 6 p^{2} P_{3 / 2}$ & 112312 & 2 & -2.306 & $4.15(+07)$ & -0.005 & -2.538 & $2.43(+07)$ & -0.004 & New \\
\hline
\end{tabular}


Table 3

(Continued)

\begin{tabular}{|c|c|c|c|c|c|c|c|c|c|c|c|c|}
\hline \multirow{2}{*}{$\begin{array}{l}\text { Line } \\
\text { Intens. }\end{array}$} & \multirow[t]{2}{*}{$\lambda(\AA)^{\mathrm{a}}$} & \multicolumn{2}{|c|}{ Designation } & \multicolumn{2}{|c|}{ Wavenumber $\left(\mathrm{cm}^{-1}\right)$} & \multicolumn{3}{|c|}{ HFR } & \multicolumn{3}{|c|}{$\mathrm{HFR}+\mathrm{CP}^{\mathrm{b}}$} & \multirow[t]{2}{*}{ Ref. $^{c}$} \\
\hline & & Lower Level & Upper Level & $\sigma_{\text {obs. }}$ & $\sigma_{\text {obs.-cal. }}$ & $\log (g f)$ & $g A\left(s^{-1}\right)$ & $\mathrm{CF}$ & $\log (g f)$ & $g A\left(s^{-1}\right)$ & $\mathrm{CF}$ & \\
\hline 3 & 894.53 & $5 s^{2} 5 d^{2} D_{3 / 2}$ & $5 s 5 p 5 d\left({ }^{3} P\right)^{2} D_{5 / 2}$ & 111791 & 2 & -2.164 & $5.71(+07)$ & -0.003 & -2.473 & $2.81(+07)$ & -0.002 & New \\
\hline 4 & 898.54 & $5 s 5 p^{2}{ }^{2} D_{5 / 2}$ & $5 p^{32} D_{5 / 2}$ & 111292 & 5 & -0.069 & $7.07(+09)$ & -0.199 & -0.258 & $4.58(+09)$ & -0.178 & New \\
\hline 7 & 899.35 & $5 s 5 p^{2}{ }^{2} P_{3 / 2}$ & $5 s^{2} 6 p^{2} P_{3 / 2}$ & 111191 & -3 & -0.691 & $1.68(+09)$ & -0.149 & -0.857 & $1.14(+09)$ & -0.134 & $*(4)$ \\
\hline 4 & 901.622 & $5 s 5 p^{2}{ }^{2} D_{5 / 2}$ & $5 p^{3}{ }^{4} S_{3 / 2}$ & 110911 & 1 & -0.620 & $1.97(+09)$ & 0.351 & -0.796 & $1.32(+09)$ & 0.328 & (7) \\
\hline 5 & 915.163 & $5 s^{2} 5 p^{2} P_{3 / 2}$ & $5 s 5 p^{2}{ }^{2} D_{3 / 2}$ & 109270 & -1 & -2.388 & $3.27(+07)$ & -0.003 & -4.163 & $5.48(+05)$ & 0.000 & (1) \\
\hline 8 & 919.57 & $5 s 5 p^{2}{ }^{2} S_{1 / 2}$ & $5 p^{3}{ }^{2} P_{3 / 2}$ & 108746 & 3 & -1.325 & $3.73(+08)$ & 0.013 & -1.551 & $2.22(+08)$ & 0.011 & New \\
\hline 7 & 928.366 & $5 s 5 p^{2}{ }^{2} D_{3 / 2}$ & $5 p^{32} D_{3 / 2}$ & 107716 & 0 & -0.649 & $1.73(+09)$ & -0.074 & -0.845 & $1.11(+09)$ & -0.066 & (7) \\
\hline 7 & 929.131 & $5 s 5 p^{2}{ }^{2} P_{3 / 2}$ & $5 p^{3}{ }^{2} P_{3 / 2}$ & 107627 & 1 & -0.362 & $3.36(+09)$ & 0.098 & -0.571 & $2.08(+09)$ & 0.082 & (7) \\
\hline 4 & 935.47 & $5 s 5 p^{2}{ }^{2} S_{1 / 2}$ & $5 s^{2} 6 p^{2} P_{1 / 2}$ & 106898 & 3 & -1.278 & $4.02(+08)$ & 0.133 & -1.464 & $2.62(+08)$ & 0.113 & New \\
\hline 7 & 942.16 & $5 s 5 p 5 d\left({ }^{3} P\right){ }^{2} P_{3 / 2}$ & $5 s^{2} 8 s^{2} S_{1 / 2}$ & 106139 & 2 & -2.850 & $1.06(+07)$ & -0.040 & -2.812 & $1.16(+07)$ & -0.043 & New \\
\hline 1 & 944.15 & $5 s^{2} 6 s^{2} S_{1 / 2}$ & $5 s 5 p 6 s\left({ }^{3} P\right){ }^{2} P_{1 / 2}$ & 105915 & -4 & -1.570 & $2.01(+08)$ & -0.150 & -1.795 & $1.20(+08)$ & -0.117 & New \\
\hline 2 & 945.18 & $5 s^{2} 5 d^{2} D_{3 / 2}$ & $5 s 5 p 5 d\left({ }^{3} P\right)^{2} D_{3 / 2}$ & 105800 & 0 & -2.104 & $5.92(+07)$ & 0.009 & -2.412 & $2.91(+07)$ & 0.006 & New \\
\hline 7 & 949.54 & $5 s^{2} 6 d^{2} D_{3 / 2}$ & $5 s^{2} 7 f^{2} F_{5 / 2}$ & 105314 & 0 & -0.421 & $2.80(+09)$ & 0.273 & -0.489 & $2.39(+09)$ & 0.271 & New \\
\hline 2 & 960.46 & $5 s^{2} 5 d^{2} D_{3 / 2}$ & $5 s 5 p 5 d\left({ }^{3} P\right){ }^{2} F_{5 / 2}$ & 104117 & 1 & -0.896 & $9.22(+08)$ & 0.048 & -1.128 & $5.40(+08)$ & 0.038 & New \\
\hline 4 & 963.18 & $5 s^{2} 6 d^{2} D_{5 / 2}$ & $5 s^{2} 7 f^{2} F_{7 / 2}$ & 103823 & -2 & -0.312 & $3.52(+09)$ & 0.246 & -0.381 & $3.00(+09)$ & 0.245 & New \\
\hline 8 & 967.55 & $5 s 5 p^{2}{ }^{2} D_{5 / 2}$ & $5 p^{32} D_{3 / 2}$ & 103354 & -2 & -0.937 & $8.28(+08)$ & 0.063 & -1.095 & $5.75(+08)$ & 0.062 & $\S(8)$ \\
\hline 3 & 970.40 & $5 s^{2} 5 d^{2} D_{5 / 2}$ & $5 s 5 p 5 d\left({ }^{3} P\right){ }^{4} D_{7 / 2}$ & 103050 & 3 & -1.688 & $1.45(+08)$ & 0.133 & -1.877 & $9.37(+07)$ & 0.119 & New \\
\hline 1 & 979.80 & $5 s^{2} 5 d^{2} D_{5 / 2}$ & $5 s 5 p 5 d\left({ }^{3} P\right){ }^{2} F_{5 / 2}$ & 102062 & 4 & -1.781 & $1.15(+08)$ & -0.018 & -1.876 & $9.26(+07)$ & -0.020 & New \\
\hline 2 & 981.21 & $5 s 5 p^{2}{ }^{2} P_{3 / 2}$ & $5 p^{3}{ }^{2} P_{1 / 2}$ & & -2 & -1.287 & & -0.0 & & & -0.039 & New \\
\hline 6 & 996.233 & $5 s^{2} 5 p^{2} P_{1 / 2}$ & $5 s 5 p^{2}{ }^{4} P_{3 / 2}$ & & 0 & -3 & $6.01(+06)$ & -0.0 & -2 & $7.02(+06)$ & -0.044 & (2) \\
\hline 5 & 997.30 & $5 s 5 p^{2}{ }^{2} P_{3 / 2}$ & $5 s 5 p 5 d\left({ }^{3} P\right){ }^{4} F_{3 / 2}$ & 1002 & -1 & -3.165 & $4.58(+06)$ & 0.004 & -3.377 & $2.81(+06)$ & 0.003 & New \\
\hline 1 & 1010.97 & $5 s^{2} 5 g^{2} G_{9 / 2}$ & $5 s^{2} 8 h^{2} H_{11 / 2}$ & 98915 & -5 & -0.388 & $2.67(+09)$ & -0.295 & -0.506 & $2.04(+09)$ & -0.286 & $*(4)$ \\
\hline 1 & 1010.97 & $5 s^{2} 5 g^{2} G_{7 / 2}$ & $5 s^{2} 8 h^{2} H_{9 / 2}$ & 98915 & 0 & -0.821 & $9.86(+08)$ & -0.138 & -0.930 & $7.67(+08)$ & -0.136 & $*(4)$ \\
\hline 1 & 1017.270 & $5 s 5 p^{2}{ }^{2} P_{1 / 2}$ & $5 p^{3}{ }^{4} S_{3 / 2}$ & 98302 & -1 & -0.923 & $7.72(+08)$ & -0.105 & -1.129 & $4.80(+08)$ & -0.091 & (7) \\
\hline 2 & 053.81 & $5 s^{2} 5 d^{2} D_{3 / 2}$ & $5 s 5 p 5 d\left({ }^{3} P\right){ }^{4} D_{3 / 2}$ & 94894 & -1 & -1.723 & $1.14(+08)$ & -0.072 & -1.920 & $7.26(+07)$ & -0.061 & New \\
\hline 6 & 057.30 & $5 s^{2} 5 f^{2} F_{7 / 2}$ & $5 s^{2} 6{ }^{2} G_{9 / 2}$ & 94581 & -4 & -0.862 & $8.24(+08)$ & 0.125 & -0.804 & $9.42(+08)$ & 0.150 & $\S(8)$ \\
\hline 3 & 1061.78 & $5 s^{2} 5 f^{2} F_{5 / 2}$ & $5 s^{2} 6 g^{2} G_{7 / 2}$ & 94181 & 4 & -0.595 & $1.50(+09)$ & 0.289 & -0.564 & $1.61(+09)$ & 0.317 & New \\
\hline 10 & 080.080 & $5 s^{2} 5 p^{2} P_{1 / 2}$ & $5 s 5 p^{2}{ }^{4} P_{1 / 2}$ & 92586 & 0 & -1.529 & $1.70(+08)$ & -0.551 & -1.687 & $1.18(+08)$ & -0.538 & (2) \\
\hline 10 & 091.634 & $5 s^{2} 5 p^{2} P_{3 / 2}$ & $5 s 5 p^{2}{ }^{4} P_{5 / 2}$ & 91606 & 0 & -1.045 & $5.03(+08)$ & -0.224 & -1.088 & $4.56(+08)$ & -0.319 & (2) \\
\hline 7 & 1101.947 & $5 s 5 p^{2}{ }^{2} P_{1 / 2}$ & $5 p^{3}{ }^{2} D_{3 / 2}$ & 90748 & 0 & -0.606 & $1.37(+09)$ & -0.092 & -0.806 & $8.62(+08)$ & -0.080 & (7) \\
\hline 1 & 10.45 & $5 s^{2} 5 d^{2} D_{3 / 2}$ & $5 s^{2} 6 p^{2} P_{3 / 2}$ & 90054 & -2 & -1.039 & $4.94(+08)$ & 0.204 & -0.934 & $6.29(+08)$ & 0.283 & New \\
\hline 2 & 1111.73 & $5 s^{2} 6 s^{2} S_{1 / 2}$ & $5 s 5 p 5 d\left({ }^{3} P\right){ }^{2} P_{1 / 2}$ & & 3 & -2 . & 3.8 & -0 & -2.276 & $2.85(+07)$ & -0.201 & $* \S(8)$ \\
\hline 2 & 1116.20 & $5 s 5 p 5 d\left({ }^{1} P\right){ }^{2} F_{7 / 2}$ & $5 s^{2} 7 d^{2} D_{5 / 2}$ & 89590 & -1 & -1.057 & $4.68(+08)$ & 0.054 & -1.202 & $3.35(+08)$ & 0.038 & New \\
\hline 8 & 1136.412 & $5 s^{2} 5 d^{2} D_{5 / 2}$ & $5 s^{2} 6 p^{2} P_{3 / 2}$ & 87996 & -2 & -0.005 & $5.10(+09)$ & 0.403 & 0.069 & $6.06(+09)$ & 0.496 & (7) \\
\hline 5 & 1144.68 & $5 s 5 p 5 d\left({ }^{1} P\right){ }^{2} P_{3 / 2}$ & $5 s^{2} 8 s^{2} S_{1 / 2}$ & 87361 & 1 & -2.242 & $2.92(+07)$ & -0.062 & -2.251 & $2.86(+07)$ & -0.060 & New \\
\hline 2 & 45.65 & $5 s 5 p 5 d\left({ }^{1} P\right){ }^{2} F_{5 / 2}$ & $5 s^{2} 7 d^{2} D_{3 / 2}$ & 87287 & -4 & -1.026 & $4.75(+08)$ & 0.084 & -1.135 & $3.70(+08)$ & 0.063 & New \\
\hline 4 & 56.25 & $5 s^{2} 5 d^{2} D_{3 / 2}$ & $5 p^{3}{ }^{2} P_{3 / 2}$ & 86486 & -3 & -1.305 & $2.48(+08)$ & 0.087 & -1.380 & $2.08(+08)$ & 0.092 & New \\
\hline 10 & 79.541 & $5 s^{2} 5 p^{2} P_{3 / 2}$ & $5 s 5 p^{2}{ }^{4} P_{3 / 2}$ & 84779 & 0 & -1.958 & $5.29(+07)$ & -0.364 & -2.084 & $3.96(+07)$ & -0.399 & (2) \\
\hline 10 & 181.465 & $5 s^{2} 5 d^{2} D_{3 / 2}$ & $5 s^{2} 6 p^{2} P_{1 / 2}$ & 84641 & 0 & -0.226 & $2.84(+09)$ & 0.442 & -0.158 & $3.32(+09)$ & 0.530 & (7) \\
\hline 6 & 181.54 & $5 s^{2} 5 d^{2} D_{5 / 2}$ & $5 s 5 p 5 d\left({ }^{3} P\right){ }^{4} F_{7 / 2}$ & 84635 & -5 & -1.149 & $3.40(+08)$ & 0.329 & -1.326 & $2.26(+08)$ & 0.311 & New \\
\hline 5 & 181.75 & $5 s 5 p^{2}{ }^{4} P_{3 / 2}$ & $5 s^{2} 4 f^{2} F_{5 / 2}$ & 84620 & 0 & -2.693 & $9.63(+06)$ & 0.486 & -2.932 & $5.55(+06)$ & 0.419 & $*(4)$ \\
\hline 5 & 1184.39 & $5 s^{2} 5 d^{2} D_{5 / 2}$ & $5 p^{3}{ }^{2} P_{3 / 2}$ & 84432 & 2 & -0.668 & $1.02(+09)$ & 0.099 & -0.677 & $1.00(+09)$ & 0.118 & New \\
\hline 5 & 202.16 & $5 s 5 p 6 s\left({ }^{3} P\right){ }^{4} P_{3 / 2}$ & $5 s^{2} 7 d^{2} D_{5 / 2}$ & & -9 & -2.976 & & -0.003 & -2.716 & $8.95(+06)$ & -0.006 & New \\
\hline 4 & 215.15 & $5 s^{2} 5 d^{2} D_{3 / 2}$ & $5 s 5 p 5 d\left({ }^{3} P\right){ }^{4} F_{5 / 2}$ & 82294 & -2 & -1.462 & $1.55(+08)$ & 0.250 & -1.645 & $1.02(+08)$ & 0.232 & New \\
\hline 7 & 1220.14 & $5 s^{2} 6 p^{2} P_{3 / 2}$ & $5 s^{2} 7 s^{2} S_{1 / 2}$ & 81958 & 4 & -0.082 & $3.71(+09)$ & -0.764 & -0.029 & $4.20(+09)$ & -0.781 & $*(5)$ \\
\hline 5 & 1228.45 & $5 s 5 p^{2}{ }^{2} P_{3 / 2}$ & $5 p^{3}{ }^{2} D_{5 / 2}$ & 81403 & -2 & -0.342 & $2.01(+09)$ & -0.103 & -0.545 & $1.26(+09)$ & -0.089 & New \\
\hline 4 & 1233.60 & $5 s 5 p 5 d\left({ }^{1} P\right){ }^{2} D_{5 / 2}$ & $5 s^{2} 7 d^{2} D_{5 / 2}$ & 81064 & 6 & -1.771 & $7.47(+07)$ & 0.093 & -1.719 & $8.42(+07)$ & 0.115 & New \\
\hline 6 & 1234.17 & $5 s 5 p^{2}{ }^{2} P_{3 / 2}$ & $5 p^{3}{ }^{4} S_{3 / 2}$ & 81026 & -2 & -1.309 & $2.14(+08)$ & 0.089 & -1.502 & $1.37(+08)$ & 0.079 & New \\
\hline 2 & 1237.84 & $5 s 5 p 5 d\left({ }^{1} P\right){ }^{2} D_{3 / 2}$ & $5 s^{2} 7 d^{2} D_{3 / 2}$ & 80786 & -3 & -2.591 & $1.17(+07)$ & 0.016 & -2.585 & $1.18(+07)$ & 0.018 & New \\
\hline 3 & 1251.50 & $5 s^{2} 5 g^{2} G_{9 / 2}$ & $5 s^{2} 7 h^{2} H_{11 / 2}$ & 79904 & 1 & 0.194 & $6.65(+09)$ & -0.450 & 0.121 & $5.62(+09)$ & -0.457 & $*(4)$ \\
\hline 2 & 253.25 & $5 s^{2} 5 g^{2} G_{7 / 2}$ & $5 s^{2} 7 h^{2} H_{9 / 2}$ & 79793 & -2 & 0.099 & $5.34(+09)$ & -0.436 & 0.025 & $4.51(+09)$ & -0.441 & $*(4)$ \\
\hline 2 & 1263.63 & $5 s^{2} 5 d^{2} D_{3 / 2}$ & $5 s 5 p 5 d\left({ }^{3} P\right){ }^{4} F_{3 / 2}$ & 79137 & 3 & -2.203 & $2.62(+07)$ & 0.061 & -2.437 & $1.53(+07)$ & 0.049 & New \\
\hline 3 & 1280.27 & $5 s 5 p^{2}{ }^{4} P_{5 / 2}$ & $5 s^{2} 4 f^{2} F_{7 / 2}$ & 78109 & -3 & -1.481 & $1.36(+08)$ & 0.477 & -1.728 & $7.68(+07)$ & 0.408 & $*(3)$ \\
\hline 3 & 1285.48 & $5 s 5 p^{2}{ }^{4} P_{5 / 2}$ & $5 s^{2} 4 f^{2} F_{5 / 2}$ & 77792 & -1 & -2.761 & $6.99(+06)$ & -0.469 & -3.005 & $3.98(+06)$ & -0.402 & $*(3)$ \\
\hline 9 & 1298.912 & $5 s^{2} 5 p^{2} P_{3 / 2}$ & $5 s 5 p^{2}{ }^{4} P_{1 / 2}$ & 76988 & 1 & -2.203 & $2.48(+07)$ & 0.107 & -2.371 & $1.69(+07)$ & 0.102 & (2) \\
\hline 5 & 1340.69 & $5 s 5 p^{2}{ }^{2} S_{1 / 2}$ & $5 p^{32} D_{3 / 2}$ & 74588 & -2 & -1.468 & $1.26(+08)$ & 0.020 & -1.665 & $8.01(+07)$ & 0.017 & $\S(8)$ \\
\hline 4 & 1361.05 & $5 s 5 p^{2}{ }^{2} P_{3 / 2}$ & $5 p^{3}{ }^{2} D_{3 / 2}$ & 73473 & -1 & -2.187 & $2.34(+07)$ & -0.003 & -2.328 & $1.69(+07)$ & -0.003 & $\S(8)$ \\
\hline 3 & 1363.66 & $5 s^{2} 6 p^{2} P_{1 / 2}$ & $5 s^{2} 6 d^{2} D_{3 / 2}$ & 73332 & 3 & 0.113 & $4.67(+09)$ & -0.438 & 0.015 & $3.73(+09)$ & -0.435 & New \\
\hline 1 & 1373.08 & $5 s 5 p 5 d\left({ }^{3} P\right){ }^{4} F_{7 / 2}$ & $5 s^{2} 6 d^{2} D_{5 / 2}$ & 72829 & -3 & -2.057 & $3.09(+07)$ & 0.111 & -2.104 & $2.77(+07)$ & 0.114 & New \\
\hline 6 & 1439.25 & $5 s^{2} 6 p^{2} P_{3 / 2}$ & $5 s^{2} 6 d^{2} D_{5 / 2}$ & 69481 & 7 & 0.269 & $5.96(+09)$ & -0.389 & 0.170 & $4.74(+09)$ & -0.384 & $*(5)$ \\
\hline 6 & 1456.37 & $5 s^{2} 7 p^{2} P_{1 / 2}$ & $5 s^{2} 8 d^{2} D_{3 / 2}$ & 68664 & 0 & -1.308 & $1.55(+08)$ & -0.150 & -1.506 & $9.80(+07)$ & -0.115 & New \\
\hline 3 & 1478.56 & $5 s^{2} 7 p^{2} P_{3 / 2}$ & $5 s^{2} 8 d^{2} D_{5 / 2}$ & 67633 & -1 & -0.713 & $5.87(+08)$ & -0.248 & -0.889 & $3.92(+08)$ & -0.210 & $*(5)$ \\
\hline 1 & 1502.59 & $5 s^{2} 6 d^{2} D_{3 / 2}$ & $5 s^{2} 6 f^{2} F_{5 / 2}$ & 66552 & 2 & 0.343 & $6.49(+09)$ & 0.641 & 0.327 & $6.26(+09)$ & 0.671 & New \\
\hline 2 & 1510.22 & $5 s 5 p 5 d\left({ }^{3} P\right){ }^{2} D_{3 / 2}$ & $5 s^{2} 7 s^{2} S_{1 / 2}$ & 66216 & 6 & -2.770 & $4.92(+06)$ & -0.107 & -2.740 & $5.28(+06)$ & -0.111 & New \\
\hline 5 & 1542.46 & $5 s^{2} 7 d^{2} D_{3 / 2}$ & $5 s^{2} 8 f^{2} F_{5 / 2}$ & 64832 & 2 & 0.048 & $3.14(+09)$ & 0.731 & 0.031 & $3.01(+09)$ & 0.740 & New \\
\hline
\end{tabular}


Table 3

(Continued)

\begin{tabular}{|c|c|c|c|c|c|c|c|c|c|c|c|c|}
\hline \multirow{2}{*}{$\begin{array}{l}\text { Line } \\
\text { Intens. }\end{array}$} & \multirow[t]{2}{*}{$\lambda(\AA)^{\mathrm{a}}$} & \multicolumn{2}{|c|}{ Designation } & \multicolumn{2}{|c|}{ Wavenumber $\left(\mathrm{cm}^{-1}\right)$} & \multicolumn{3}{|c|}{ HFR } & \multicolumn{3}{|c|}{$\mathrm{HFR}+\mathrm{CP}^{\mathrm{b}}$} & \multirow[t]{2}{*}{ Ref. $^{c}$} \\
\hline & & Lower Level & Upper Level & $\sigma_{\text {obs. }}$ & $\sigma_{\text {obs. }- \text { cal. }}$ & $\log (g f)$ & $g A\left(s^{-1}\right)$ & $\mathrm{CF}$ & $\log (g f)$ & $g A\left(s^{-1}\right)$ & $\mathrm{CF}$ & \\
\hline 2 & 1558.56 & $5 s^{2} 7 d^{2} D_{5 / 2}$ & $5 s^{2} 8 f^{2} F_{7 / 2}$ & 64162 & 3 & 0.020 & $2.88(+09)$ & 0.787 & 0.002 & $2.76(+09)$ & 0.798 & New \\
\hline 7 & 1663.09 & $5 s 5 p^{2}{ }^{2} D_{3 / 2}$ & $5 s^{2} 4 f^{2} F_{5 / 2}$ & 60129 & 1 & -0.815 & $3.65(+08)$ & -0.461 & -1.095 & $1.92(+08)$ & -0.383 & $*(3)$ \\
\hline 10 & 1782.95 & $5 s 5 p^{2}{ }^{2} D_{5 / 2}$ & $5 s^{2} 4 f^{2} F_{7 / 2}$ & 56087 & 0 & -0.720 & $4.05(+08)$ & -0.436 & -1.003 & $2.11(+08)$ & -0.360 & $*(3)$ \\
\hline 4 & 1793.03 & $5 s 5 p^{2}{ }^{2} D_{5 / 2}$ & $5 s^{2} 4 f^{2} F_{5 / 2}$ & 55772 & 4 & -1.998 & $2.09(+07)$ & 0.433 & -2.277 & $1.10(+07)$ & 0.359 & * (3) \\
\hline 5 & 1857.13 & $5 s 5 p 5 d\left({ }^{3} P\right)^{2} F_{5 / 2}$ & $5 s^{2} 6 d^{2} D_{3 / 2}$ & 53847 & -7 & -3.894 & $2.47(+05)$ & 0.000 & -4.174 & $1.30(+05)$ & 0.000 & New \\
\hline 6 & 1864.10 & $5 s^{2} 6 f^{2} F_{5 / 2}$ & $5 s^{2} 7 g^{2} G_{7 / 2}$ & 53645 & -2 & -1.842 & $2.76(+07)$ & -0.113 & -2.161 & $1.32(+07)$ & -0.077 & New \\
\hline 7 & 1867.63 & $5 s^{2} 6 f^{2} F_{7 / 2}$ & $5 s^{2} 7 g^{2} G_{9 / 2}$ & 53544 & 2 & -1.663 & $4.17(+07)$ & -0.119 & -1.954 & $2.14(+07)$ & -0.085 & New \\
\hline 7 & 1976.97 & $5 s^{2} 5 g^{2} G_{9 / 2}$ & $5 s^{2} 6 h^{2} H_{11 / 2}$ & 50582 & 0 & 1.183 & $2.61(+10)$ & -0.868 & 1.154 & $2.43(+10)$ & -0.883 & * (4) \\
\hline 8 & 1977.09 & $5 s^{2} 5 g^{2} G_{7 / 2}$ & $5 s^{2} 6 h^{2} H_{9 / 2}$ & 50579 & 1 & 1.094 & $2.11(+10)$ & -0.874 & 1.064 & $1.97(+10)$ & -0.889 & $*(4)$ \\
\hline 2 & 2056.72 & $5 s^{2} 6 h^{2} H_{9 / 2}$ & $5 s^{2} 8 i^{2} I_{11 / 2}$ & 48621 & -1 & 0.357 & $3.59(+09)$ & 0.892 & 0.323 & $3.32(+09)$ & 0.889 & * (4) \\
\hline 2 & 2056.80 & $5 s^{2} 6 h^{2} H_{11 / 2}$ & $5 s^{2} 8 i^{2} I_{13 / 2}$ & 48619 & 1 & 0.431 & $4.25(+09)$ & 0.904 & 0.397 & $3.93(+09)$ & 0.900 & *(4) \\
\hline 2 & 2094.78 & $5 s 5 p 5 d\left({ }^{3} P\right){ }^{2} D_{5 / 2}$ & $5 s^{2} 6 d^{2} D_{5 / 2}$ & 47738 & -3 & -2.560 & $4.16(+06)$ & 0.008 & -2.558 & $4.18(+06)$ & 0.009 & New \\
\hline \multicolumn{13}{|c|}{ Air wavelength } \\
\hline 2 & 2204.20 & $5 s^{2} 5 f^{2} F_{7 / 2}$ & $5 s^{2} 5 g^{2} G_{9 / 2}$ & 45354 & 1 & 0.816 & $9.00(+09)$ & -0.646 & 0.776 & $8.22(+09)$ & -0.673 & New \\
\hline 4 & 2219.25 & $5 s^{2} 5 f^{2} F_{5 / 2}$ & $5 s^{2} 5 g^{2} G_{7 / 2}$ & 45046 & -4 & 0.670 & $6.35(+09)$ & -0.637 & 0.631 & $5.80(+09)$ & -0.664 & $*(4)$ \\
\hline 5 & 2310.79 & $5 s^{2} 6 s^{2} S_{1 / 2}$ & $5 p^{3}{ }^{2} P_{3 / 2}$ & 43262 & 1 & -0.526 & $3.73(+08)$ & 0.467 & -0.647 & $2.82(+08)$ & 0.454 & New \\
\hline 12 & 2413.84 & $5 s^{2} 6 s^{2} S_{1 / 2}$ & $5 s^{2} 6 p^{2} P_{1 / 2}$ & 41415 & 2 & -0.202 & $7.18(+08)$ & -0.573 & -0.341 & $5.22(+08)$ & -0.566 & $*(5)$ \\
\hline 5 & 2530.47 & $5 s 5 p 6 s\left({ }^{1} P\right){ }^{2} P_{3 / 2}$ & $5 s^{2} 7 d^{2} D_{5 / 2}$ & 39506 & 0 & 0.309 & $2.13(+09)$ & 0.623 & 0.286 & $2.02(+09)$ & 0.646 & $* \S(5)$ \\
\hline 1 & 2533.78 & $5 s 5 p 5 d\left({ }^{3} P\right)^{2} F_{7 / 2}$ & $5 s^{2} 6 d^{2} D_{5 / 2}$ & 39455 & -3 & -4.388 & $4.19(+04)$ & 0.000 & -4.064 & $8.83(+04)$ & 0.000 & New \\
\hline 4 & 2574.08 & $5 s 5 p 5 d\left({ }^{3} P\right){ }^{2} P_{1 / 2}$ & $5 s^{2} 7 s^{2} S_{1 / 2}$ & 38837 & 2 & -2.220 & $6.09(+06)$ & 0.297 & -2.226 & $6.00(+06)$ & 0.292 & New \\
\hline 1 & 2578.62 & $5 s^{2} 7 d^{2} D_{5 / 2}$ & $5 s^{2} 7 f^{2} F_{7 / 2}$ & 38769 & -6 & 0.731 & $5.36(+09)$ & 0.805 & 0.726 & $5.30(+09)$ & 0.807 & New \\
\hline 7 & 2622.96 & $5 s^{2} 7 p^{2} P_{3 / 2}$ & $5 s^{2} 8 s^{2} S_{1 / 2}$ & 38113 & 0 & 0.140 & $1.34(+09)$ & -0.945 & 0.158 & $1.40(+09)$ & -0.949 & New \\
\hline 4 & 2798.03 & $5 s^{2} 6 d^{2} D_{3 / 2}$ & $5 s^{2} 7 p^{2} P_{1 / 2}$ & 35729 & 0 & -0.168 & $5.73(+08)$ & 0.661 & -0.153 & $5.93(+08)$ & 0.688 & * (5) \\
\hline 4 & 2798.58 & $5 s^{2} 6 d^{2} D_{5 / 2}$ & $5 s^{2} 7 p^{2} P_{3 / 2}$ & 35722 & 2 & 0.154 & $1.22(+09)$ & 0.745 & 0.168 & $1.27(+09)$ & 0.768 & $*(5)$ \\
\hline $10 \mathrm{w}$ & 3259.98 & $5 s^{2} 6 g^{2} G_{7 / 2}$ & $5 s^{2} 7 h^{2} H_{9 / 2}$ & 30666 & -2 & 1.048 & $7.08(+09)$ & -0.941 & 1.038 & $6.91(+09)$ & -0.947 & * (4) \\
\hline $10 \mathrm{w}$ & 3259.98 & $5 s^{2} 6 g^{2} G_{9 / 2}$ & $5 s^{2} 7 h^{2} H_{11 / 2}$ & 30666 & -5 & 0.999 & $6.18(+09)$ & -0.912 & 0.988 & $6.03(+09)$ & -0.918 & * (4) \\
\hline 4 & 3309.29 & $5 s^{2} 7 p^{2} P_{1 / 2}$ & $5 s^{2} 7 d^{2} D_{3 / 2}$ & 30209 & -1 & 0.244 & $1.06(+09)$ & -0.550 & 0.211 & $9.81(+08)$ & -0.551 & New \\
\hline 12 & 3366.26 & $5 s^{2} 6 h^{2} H_{9 / 2}$ & $5 s^{2} 7 i^{2} I_{11 / 2}$ & 29698 & 0 & 1.291 & $1.15(+10)$ & 0.961 & 1.278 & $1.12(+10)$ & 0.961 & $*(4)$ \\
\hline 12 & 3366.60 & $5 s^{2} 6 h^{2} H_{11 / 2}$ & $5 s^{2} 7 i^{2} I_{13 / 2}$ & 29695 & 1 & 1.366 & $1.37(+10)$ & 0.965 & 1.353 & $1.33(+10)$ & 0.965 & * (4) \\
\hline 3 & 3408.52 & $5 s^{2} 7 p^{2} P_{3 / 2}$ & $5 s^{2} 7 d^{2} D_{5 / 2}$ & 29330 & 0 & 0.626 & $2.45(+09)$ & -0.707 & 0.594 & $2.27(+09)$ & -0.708 & New \\
\hline 9 & 3586.04 & $5 s^{2} 5 g^{2} G_{9 / 2}$ & $5 s^{2} 6 f^{2} F_{7 / 2}$ & 27878 & -2 & 0.235 & $8.87(+08)$ & 0.886 & 0.238 & $8.92(+08)$ & 0.900 & New \\
\hline 12 & 3599.49 & $5 s^{2} 5 g^{2} G_{7 / 2}$ & $5 s^{2} 6 f^{2} F_{5 / 2}$ & 27774 & -1 & 0.179 & $7.81(+08)$ & 0.931 & 0.181 & $7.83(+08)$ & 0.935 & New \\
\hline 3 & 3790.22 & $5 s^{2} 8 d^{2} D_{3 / 2}$ & $5 s^{2} 8 f^{2} F_{5 / 2}$ & 26376 & 0 & 0.473 & $1.37(+09)$ & 0.853 & 0.471 & $1.36(+09)$ & 0.856 & New \\
\hline 3 & 3843.97 & $5 s 5 p 5 d\left({ }^{1} P\right){ }^{2} P_{3 / 2}$ & $5 s^{2} 7 s^{2} S_{1 / 2}$ & 26007 & 0 & -1.974 & $4.80(+06)$ & -0.438 & -2.008 & $4.44(+06)$ & -0.394 & New \\
\hline 3 & 3861.93 & $5 s 5 p^{2}{ }^{2} P_{3 / 2}$ & $5 s^{2} 4 f^{2} F_{5 / 2}$ & 25886 & 0 & -2.161 & $3.03(+06)$ & 0.424 & -2.521 & $1.32(+06)$ & 0.334 & * (3) \\
\hline 2 & 3866.13 & $5 s^{2} 8 d^{2} D_{5 / 2}$ & $5 s^{2} 8 f^{2} F_{7 / 2}$ & 25858 & 3 & 0.646 & $2.03(+09)$ & 0.851 & 0.644 & $2.02(+09)$ & 0.855 & New \\
\hline 10 & 4301.10 & $5 s^{2} 7 s^{2} S_{1 / 2}$ & $5 s^{2} 7 p^{2} P_{3 / 2}$ & 23243 & 3 & 0.310 & $7.36(+08)$ & 0.881 & 0.268 & $6.68(+08)$ & 0.875 & $*(5)$ \\
\hline 2 & 4681.53 & $5 s^{2} 6 f^{2} F_{5 / 2}$ & $5 s^{2} 6 g^{2} G_{7 / 2}$ & 21355 & 3 & 0.809 & $1.93(+09)$ & -0.864 & 0.795 & $1.86(+09)$ & -0.878 & New \\
\hline 3 & 4681.65 & $5 s^{2} 6 f^{2} F_{7 / 2}$ & $5 s^{2} 6 g^{2} G_{9 / 2}$ & 21354 & 2 & 0.748 & $1.74(+09)$ & -0.842 & 0.733 & $1.68(+09)$ & -0.856 & New \\
\hline 4 & 5113.09 & $5 s^{2} 8 p^{2} P_{1 / 2}$ & $5 s^{2} 8 d^{2} D_{3 / 2}$ & 19552 & -35 & 0.394 & $6.36(+08)$ & -0.930 & 0.382 & $6.18(+08)$ & -0.929 & New \\
\hline 6 & 5151.79 & $5 s^{2} 7 h^{2} H_{9 / 2}$ & $5 s^{2} 8 i^{2} I_{11 / 2}$ & 19405 & 0 & 1.228 & $4.23(+09)$ & 0.902 & 1.223 & $4.18(+09)$ & 0.902 & New \\
\hline 2 & 5234.65 & $5 s^{2} 7 i^{2} I_{11 / 2}$ & $5 s^{2} 8 k^{2} K_{13 / 2}$ & 19098 & 0 & 1.451 & $6.88(+09)$ & -1.000 & 1.445 & $6.78(+09)$ & -1.000 & $*(4)$ \\
\hline 2 & 5234.65 & $5 s^{2} 7 i^{2} I_{13 / 2}$ & $5 s^{2} 8 k^{2} K_{15 / 2}$ & 19098 & 0 & 1.514 & 7.94(+09) & -1.000 & 1.508 & $7.84(+09)$ & -1.000 & $*(4)$ \\
\hline 2 & 5281.57 & $5 s^{2} 8 p^{2} P_{3 / 2}$ & $5 s^{2} 8 d^{2} D_{5 / 2}$ & 18928 & 24 & 0.820 & $1.53(+09)$ & -0.925 & 0.808 & $1.49(+09)$ & -0.925 & * (5) \\
\hline 2 & 5288.04 & $5 s^{2} 7 d^{2} D_{3 / 2}$ & $5 s^{2} 8 p^{2} P_{1 / 2}$ & 18905 & 38 & 0.183 & $3.66(+08)$ & 0.795 & 0.190 & $3.71(+08)$ & 0.800 & New \\
\hline 10 & 5714.56 & $5 s^{2} 7 g^{2} G_{9 / 2}$ & $5 s^{2} 8 h^{2} H_{11 / 2}$ & 17494 & -4 & 1.030 & $2.18(+09)$ & -0.946 & 1.026 & $2.16(+09)$ & -0.947 & New \\
\hline 10 & 5714.56 & $5 s^{2} 7 g^{2} G_{7 / 2}$ & $5 s^{2} 8 h^{2} H_{9 / 2}$ & 17494 & 1 & 0.718 & $1.07(+09)$ & -0.911 & 0.714 & $1.06(+09)$ & -0.914 & New \\
\hline 1 & 5741.75 & $5 s^{2} 6 g^{2} G_{7 / 2}$ & $5 s^{2} 7 f^{2} F_{5 / 2}$ & 17411 & -1 & 0.557 & $7.36(+08)$ & 0.975 & 0.559 & $7.40(+08)$ & 0.977 & New \\
\hline 1 & 5753.14 & $5 s^{2} 6 g^{2} G_{9 / 2}$ & $5 s^{2} 7 f^{2} F_{7 / 2}$ & 17377 & -1 & 0.545 & $6.97(+08)$ & 0.975 & 0.547 & $6.99(+08)$ & 0.976 & New \\
\hline
\end{tabular}

\section{Notes.}

* Revised wavelength.

$\S$ Revised classification.

$\dagger$ Classification revised by Saloman (2004).

$\ddagger$ For conversion from air wavelength to vacuum we used the dispersion formula from Edlén (1966).

${ }^{a}$ Uncertainty: New and revised wavelengths below 2100 A: $0.02 \AA$; New and revised wavelengths above 2100 Å: $0.01 \AA$ A Kaufman \& Sugar (1987); Tauheed et al. (1992); Churilov \& Joshi (2000): $0.005 \AA$; Reyna Almandos et al. (2001): $0.02 \AA$.

${ }^{\mathrm{b}}$ Hartree-Fock relativistic + core polarization: for $5 s^{2} n l-5 s^{2} n^{\prime} l^{\prime}$ transitions $\alpha=5.80 a_{0}{ }^{3}$; for transitions involving unfilled $5 s$ subshell, $\alpha=0.993 a_{0}{ }^{3}$.

${ }^{c}$ References: (1) Kaufman \& Sugar 1987; (2) Tauheed et al. 1992; (3) Larsson et al. 1996; (4) Wang et al. 1996; (5) Wang et al. 1997a; (6) Wang et al. 1997b; (7) Churilov \& Joshi 2000; (8) Reyna Almandos et al. 2001. 
Table 4

Energy Levels and Lifetimes of Xe VI

\begin{tabular}{|c|c|c|c|c|c|c|c|c|c|}
\hline \multirow[t]{2}{*}{ Designation } & \multirow[t]{2}{*}{ LSJ } & \multirow{2}{*}{$\begin{array}{c}\text { Exp. } \\
\text { Values } \\
\left(\mathrm{cm}^{-1}\right)\end{array}$} & \multirow{2}{*}{$\begin{array}{l}\text { Fitted } \\
\left(\mathrm{cm}^{-1}\right)\end{array}$} & \multirow[t]{2}{*}{ Lin. } & \multirow[t]{2}{*}{ Composition $^{\mathrm{a}}$} & \multicolumn{3}{|c|}{ Lifetime (ns) } & \multirow[b]{2}{*}{ Ref. $^{\circ}$} \\
\hline & & & & & & $\begin{array}{l}\text { Without } \\
\mathrm{CP}\end{array}$ & $\mathrm{HFR}+\mathrm{CP}^{\mathrm{b}}$ & $\begin{array}{l}\text { Exp. } \\
\text { Beam } \\
\text { Foil }^{\mathrm{c}}\end{array}$ & \\
\hline \multirow[t]{2}{*}{$5 s^{2} 5 p$} & ${ }^{2} P_{1 / 2}$ & 0 & 1 & 8 & $98 \%{ }^{2} P$ & & & & \\
\hline & ${ }^{2} P_{3 / 2}$ & 15599 & 15598 & 11 & $97 \%{ }^{2} P$ & & & & (7) \\
\hline \multirow[t]{2}{*}{$5 s^{2} 4 f$} & ${ }^{2} F_{5 / 2}$ & 184998 & 184814 & 6 & $96 \%{ }^{2} F$ & 14.774 & 28.083 & & $*(3)$ \\
\hline & ${ }^{2} F_{7 / 2}$ & 185317 & 185496 & 3 & $96 \%{ }^{2} F$ & 14.790 & 27.801 & & $*(3)$ \\
\hline \multirow[t]{5}{*}{$5 p^{3}$} & ${ }^{2} D_{3 / 2}$ & 232585.5 & 232639 & 8 & $36 \%{ }^{2} D+33 \% 5 p^{3}{ }^{4} S+18 \% 5 p^{3}{ }^{2} P+9 \% 5 s 5 p\left({ }^{3} P\right) 5 d^{2} D$ & 0.163 & 0.240 & & (7) \\
\hline & ${ }^{4} S_{3 / 2}$ & 240140.0 & 240072 & 6 & $58 \%{ }^{4} S+31 \% 5 p^{3}{ }^{2} D+10 \% 5 s 5 p\left({ }^{3} P\right) 5 d^{2} D$ & 0.115 & 0.169 & $0.12 \pm 0.02$ & (7) \\
\hline & ${ }^{2} D_{5 / 2}$ & 240517 & 240535 & 4 & $77 \%{ }^{2} D+22 \% 5 s 5 p\left({ }^{3} P\right) 5 d^{2} D$ & 0.514 & 0.804 & & New \\
\hline & ${ }^{2} P_{1 / 2}$ & 261029 & 260963 & 4 & $72 \%{ }^{2} P+12 \% 5 s^{2} 6 p^{2} P+10 \% 5 s 5 p\left({ }^{3} P\right) 5 d^{2} P$ & 0.197 & 0.303 & & New \\
\hline & ${ }^{2} P_{3 / 2}$ & 266738.3 & 266830 & 9 & $46 \%{ }^{2} P+19 \% 5 s^{2} 6 p^{2} P+12 \% 5 s 5 p\left({ }^{3} P\right) 5 d^{2} P+7 \% 5 p^{3}{ }^{4} S+6 \% 5 p^{3}{ }^{2} D$ & 0.237 & 0.360 & & (7) \\
\hline \multirow[t]{2}{*}{$5 s^{2} 6 p$} & ${ }^{2} P_{1 / 2}$ & 264891.0 & 264900 & 7 & $83 \%{ }^{2} P+12 \% 5 p^{3}{ }^{2} P$ & 0.220 & 0.227 & $0.20 \pm 0.02$ & (7) \\
\hline & ${ }^{2} P_{3 / 2}$ & 270305.7 & 270280 & 10 & $75 \%{ }^{2} P+15 \% 5 p^{3}{ }^{2} P$ & 0.187 & 0.198 & & (7) \\
\hline \multirow{17}{*}{$5 s 5 p\left({ }^{3} P\right) 5 d$} & ${ }^{4} F_{3 / 2}$ & 259384 & 259391 & 5 & $93 \%{ }^{4} F$ & 1.314 & 1.746 & & New \\
\hline & ${ }^{4} F_{5 / 2}$ & 262545.9 & 262348 & 3 & $91 \%{ }^{4} F$ & 0.855 & 1.108 & & (7) \\
\hline & ${ }^{4} F_{7 / 2}$ & 266948 & 267005 & 4 & $89 \%{ }^{4} F+8 \% 5 s 5 p\left({ }^{3} P\right) 5 d{ }^{4} D$ & 0.631 & 0.805 & & New \\
\hline & ${ }^{4} P_{5 / 2}$ & 273614.0 & 273647 & 4 & $56 \%{ }^{4} P+28 \% 5 s 5 p\left({ }^{3} P\right) 5 d^{4} D+7 \% 5 s 5 p\left({ }^{3} P\right) 5 d^{2} D+6 \% 5 s 5 p\left({ }^{1} P\right) 5 d^{2} D$ & 0.061 & 0.078 & & (7) \\
\hline & ${ }^{4} F_{9 / 2}$ & & [275 328] & & $99 \%{ }^{4} F$ & $108 \mu \mathrm{s}$ & $172 \mu \mathrm{s}$ & & * \\
\hline & ${ }^{4} D_{3 / 2}$ & 275145 & 275326 & 5 & $61 \%{ }^{4} D+30 \% 5 s 5 p\left({ }^{3} P\right) 5 d{ }^{4} P$ & 0.046 & 0.059 & & New \\
\hline & ${ }^{4} D_{1 / 2}$ & 276225 & 276078 & 4 & $86 \%{ }^{4} D+8 \% 5 s 5 p\left({ }^{3} P\right) 5 d^{4} P$ & 0.039 & 0.050 & & New \\
\hline & ${ }^{2} F_{5 / 2}$ & 284366.0 & 284485 & 8 & $35 \%{ }^{2} F+20 \% 5 s 5 p\left({ }^{3} P\right) 5 d^{2} D+15 \% 5 s 5 p\left({ }^{3} P\right) 5 d^{4} D+15 \% 5 s 5 p\left({ }^{1} P\right) 5 d^{2} F+7 \% 5 p^{3}{ }^{2} D$ & 0.074 & 0.097 & & (7) \\
\hline & ${ }^{4} D_{7 / 2}$ & 285355 & 285227 & 3 & $89 \%{ }^{4} D+8 \% 5 s 5 p\left({ }^{3} P\right) 5 d{ }^{4} F$ & 0.046 & 0.058 & & New \\
\hline & ${ }^{2} D_{3 / 2}$ & 286050 & 286359 & 7 & $37 \%{ }^{2} D+21 \% 5 s 5 p\left({ }^{3} P\right) 5 d^{4} D+17 \% 5 s 5 p\left({ }^{3} P\right) 5 d^{4} P+10 \% 5 p^{3}{ }^{2} D+6 \% 5 s 5 p\left({ }^{1} P\right) 5 d^{2} D$ & 0.050 & 0.066 & & New \\
\hline & ${ }^{4} D_{5 / 2}$ & 286688 & 286671 & 3 & $51 \%{ }^{4} D+29 \% 5 s 5 p\left({ }^{3} P\right) 5 d{ }^{4} P+13 \% 5 s 5 p\left({ }^{3} P\right) 5 d{ }^{2} F$ & 0.052 & 0.066 & & New \\
\hline & ${ }^{4} P_{1 / 2}$ & 287170 & 287180 & 4 & $90 \%{ }^{4} P+9 \% 5 s 5 p\left({ }^{3} P\right) 5 d^{4} D$ & 0.052 & 0.066 & & New \\
\hline & ${ }^{4} P_{3 / 2}$ & 287804 & 287674 & 5 & $50 \%{ }^{4} P+23 \% 5 s 5 p\left({ }^{3} P\right) 5 d^{2} D+13 \% 5 s 5 p\left({ }^{3} P\right) 5 d^{4} D+6 \% 5 p^{3}{ }^{2} D$ & 0.055 & 0.071 & & New \\
\hline & ${ }^{2} D_{5 / 2}$ & 292038.4 & 292026 & 5 & $35 \%^{2} D+22 \% 5 s 5 p\left({ }^{3} P\right) 5 d^{2} F+14 \% 5 s 5 p\left({ }^{1} P\right) 5 d^{2} D+14 \% 5 s 5 p\left({ }^{3} P\right) 5 d^{4} P+6 \% 5 s 5 p\left({ }^{1} P\right) 5 d^{2} F+5 \% 5 p^{3}{ }^{2} D$ & 0.077 & 0.101 & & $*(7)$ \\
\hline & ${ }^{2} F_{7 / 2}$ & 300322 & 300440 & 2 & $72 \%{ }^{2} F+24 \% 5 s 5 p\left({ }^{1} P\right) 5 d^{2} F$ & 0.108 & 0.139 & & New \\
\hline & ${ }^{2} P_{3 / 2}$ & 307476 & 307305 & 5 & $58 \%{ }^{2} P+24 \% 5 s 5 p\left({ }^{1} P\right) 5 d^{2} D+11 \% 5 p^{3}{ }^{2} P$ & 0.041 & 0.054 & & New \\
\hline & ${ }^{2} P_{1 / 2}$ & 313425 & 313362 & 8 & $74 \%{ }^{2} P+7 \% 5 s 5 p\left({ }^{3} P\right) 6 s^{2} P+6 \% 5 p^{3}{ }^{2} P+5 \% 5 s 5 p\left({ }^{1} P\right) 5 d^{2} P$ & 0.058 & 0.074 & & New \\
\hline \multirow[t]{6}{*}{$5 s 5 p\left({ }^{1} P\right) 5 d$} & ${ }^{2} F_{7 / 2}$ & 315238.6 & 315518 & 2 & $53 \%{ }^{2} F+21 \% 5 s 5 p\left({ }^{3} P\right) 5 d^{2} F+19 \% 5 s^{2} 5 f^{2} F$ & 0.053 & 0.067 & & (7) \\
\hline & ${ }^{2} F_{5 / 2}$ & 316868 & 317016 & 4 & $49 \%{ }^{2} F+24 \% 5 s^{2} 5 f^{2} F+17 \% 5 s 5 p\left({ }^{3} P\right) 5 d^{2} F$ & 0.052 & 0.065 & & New \\
\hline & ${ }^{2} D_{3 / 2}$ & 323370 & 323266 & 4 & $29 \%{ }^{2} D+43 \% 5 s 5 p\left({ }^{3} P\right) 6 s{ }^{4} P+11 \% 5 s 5 p\left({ }^{3} P\right) 5 d^{2} P+5 \% 5 s 5 p\left({ }^{3} P\right) 5 d^{2} D$ & 0.043 & 0.050 & & New \\
\hline & ${ }^{2} D_{5 / 2}$ & 323772 & 323703 & 4 & $70 \%{ }^{2} D+12 \% 5 s 5 p\left({ }^{3} P\right) 5 d^{2} D+6 \% 5 p^{3}{ }^{2} D$ & 0.032 & 0.042 & & New \\
\hline & ${ }^{2} P_{1 / 2}$ & 324045 & 323987 & 2 & $82 \%{ }^{2} P+6 \% 5 p^{3}{ }^{2} P$ & 0.037 & 0.052 & & $*(7)$ \\
\hline & ${ }^{2} P_{3 / 2}$ & 326253 & 326221 & 6 & $74 \%{ }^{2} P+10 \% 5 s 5 p\left({ }^{3} P\right) 6 s{ }^{4} P$ & 0.042 & 0.057 & & New \\
\hline \multirow[t]{2}{*}{$5 s^{2} 5 f$} & ${ }^{2} F_{7 / 2}$ & 331642 & 331574 & 3 & $77 \%{ }^{2} F+17 \% 5 s 5 p\left({ }^{1} P\right) 5 d^{2} F$ & 0.050 & 0.065 & $0.06 \pm 0.02$ & $*(4)$ \\
\hline & ${ }^{2} F_{5 / 2}$ & 331945 & 331900 & 3 & $70 \%{ }^{2} F+21 \% 5 s 5 p\left({ }^{1} P\right) 5 d^{2} F$ & 0.043 & 0.055 & $0.05 \pm 0.02$ & $*(4)$ \\
\hline \multirow[t]{5}{*}{$5 s 5 p\left({ }^{3} P\right) 6 s$} & ${ }^{4} P_{1 / 2}$ & 318344 & 318497 & 2 & $91 \%{ }^{4} P$ & 0.103 & 0.090 & & New \\
\hline & ${ }^{4} P_{3 / 2}$ & 321637 & 321478 & 5 & $35 \%{ }^{4} P+27 \% 5 s 5 p\left({ }^{1} P\right) 5 d^{2} D+14 \% 5 s 5 p\left({ }^{1} P\right) 5 d^{2} P+7 \% 5 s 5 p\left({ }^{3} P\right) 5 d^{2} P+6 \% 5 s 5 p\left({ }^{3} P\right) 6 s^{2} P$ & 0.054 & 0.064 & & New \\
\hline & ${ }^{2} P_{1 / 2}$ & 329397 & 329362 & 4 & $84 \%{ }^{2} P+5 \% 5 s 5 p\left({ }^{1} P\right) 6 s^{2} P$ & 0.052 & 0.050 & & New \\
\hline & ${ }^{4} P_{5 / 2}$ & & [337006] & & $99 \%{ }^{4} P$ & 0.095 & 0.080 & & * \\
\hline & ${ }^{2} P_{3 / 2}$ & 344230 & 344274 & 2 & $86 \%{ }^{2} P+8 \% 5 s 5 p\left({ }^{3} P\right) 6 s^{4} P$ & 0.046 & 0.043 & & New \\
\hline \multirow[t]{2}{*}{$5 s 5 p\left({ }^{1} P\right) 6 s$} & ${ }^{2} P_{1 / 2}$ & 363623 & 363619 & 3 & $61 \%^{2} P+33 \% 5 s^{2} 7 p^{2} P$ & 0.057 & 0.056 & & New \\
\hline & ${ }^{2} P_{3 / 2}$ & 365324 & 365337 & 3 & $75 \%{ }^{2} P+17 \% 5 s^{2} 7 p^{2} P$ & 0.056 & 0.058 & & New \\
\hline \multirow[t]{2}{*}{$5 s^{2} 7 p$} & ${ }^{2} P_{1 / 2}$ & 373949 & 373951 & 3 & $63 \%{ }^{2} P+30 \% 5 s 5 p\left({ }^{1} P\right) 6 s^{2} P$ & 0.093 & 0.102 & & $*(5)$ \\
\hline & ${ }^{2} P_{3 / 2}$ & 375500 & 375500 & 5 & $79 \%{ }^{2} P+17 \% 5 s 5 p\left({ }^{1} P\right) 6 s{ }^{2} P$ & 0.153 & 0.166 & & (5) \\
\hline $5 s^{2} 6 f$ & ${ }^{2} F_{5 / 2}$ & 404770 & 404843 & 5 & $84 \%{ }^{2} F+6 \% 5 p^{2}\left({ }^{3} P\right) 4 f^{2} D$ & 0.122 & 0.170 & & New \\
\hline & ${ }^{2} F_{7 / 2}$ & 404875 & 404789 & 3 & $73 \%^{2} F+8 \% 5 p^{2}\left({ }^{1} D\right) 4 f^{2} F+7 \% 5 p^{2}\left({ }^{3} P\right) 4 f^{4} D+5 \% 5 p^{2}\left({ }^{3} P\right) 4 f^{4} G$ & 0.137 & 0.195 & & New \\
\hline $5 s^{2} 8 p$ & ${ }^{2} P_{1 / 2}$ & 423026 & 422992 & 2 & $97 \%^{2} P$ & 0.768 & 0.556 & & $*(5)$ \\
\hline & ${ }^{2} P_{3 / 2}$ & 424230 & 424282 & 1 & $94 \%{ }^{2} P$ & 0.744 & 0.548 & & $*(5)$ \\
\hline $5 s^{2} 6 h$ & ${ }^{2} H_{11 / 2}$ & 427577 & 427595 & 3 & $97 \%{ }^{2} \mathrm{H}$ & 0.420 & 0.454 & & $*(4)$ \\
\hline
\end{tabular}


Table 4

\begin{tabular}{|c|c|c|c|c|c|c|c|c|c|}
\hline \multirow[t]{2}{*}{ Designation } & \multirow[t]{2}{*}{ LSJ } & \multirow{2}{*}{$\begin{array}{c}\text { Exp. } \\
\text { Values } \\
\left(\mathrm{cm}^{-1}\right)\end{array}$} & \multirow{2}{*}{$\begin{array}{l}\text { Fitted } \\
\left(\mathrm{cm}^{-1}\right)\end{array}$} & \multirow[t]{2}{*}{ Lin. } & \multirow[t]{2}{*}{ Composition $^{\mathrm{a}}$} & \multicolumn{3}{|c|}{ Lifetime (ns) } & \multirow[b]{2}{*}{ Ref. } \\
\hline & & & & & & $\begin{array}{l}\text { Without } \\
\mathrm{CP}\end{array}$ & $\mathrm{HFR}+\mathrm{CP}^{\mathrm{b}}$ & $\begin{array}{l}\text { Exp. } \\
\text { Beam } \\
\text { Foil }^{c}\end{array}$ & \\
\hline & ${ }^{2} H_{9 / 2}$ & 427573 & 427559 & 3 & $97 \%{ }^{2} H$ & 0.414 & 0.449 & & $*(4)$ \\
\hline \multirow[t]{2}{*}{$5 s 27 f$} & ${ }^{2} F_{5 / 2}$ & 443534 & 443517 & 2 & $95 \%{ }^{2} F$ & 0.199 & 0.303 & & New \\
\hline & ${ }^{2} F_{7 / 2}$ & 443605 & 443625 & 3 & $96 \%^{2} F$ & 0.209 & 0.320 & & New \\
\hline \multirow{2}{*}{$5 s^{2} 7 h$} & ${ }^{2} H_{9 / 2}$ & 456790 & 456834 & 3 & $96 \%{ }^{2} H$ & 0.678 & 0.746 & & $*(4)$ \\
\hline & ${ }^{2} H_{11 / 2}$ & 456898 & 456852 & 2 & $97 \%{ }^{2} \mathrm{H}$ & 0.679 & 0.744 & & $*(4)$ \\
\hline \multirow{2}{*}{$5 s^{2} 8 f$} & ${ }^{2} F_{5 / 2}$ & 468989 & 468884 & 2 & $94 \%^{2} F$ & 0.272 & 0.396 & & New \\
\hline & ${ }^{2} F_{7 / 2}$ & 468989 & 469158 & 2 & $62 \%{ }^{2} F+16 \% 5 s 5 p\left({ }^{3} P\right) 5 g^{4} H+7 \% 5 s 5 p\left({ }^{3} P\right) 5{ }^{4} G$ & 0.145 & 0.179 & & New \\
\hline \multirow[t]{2}{*}{$5 s^{2} 8 h$} & ${ }^{2} H_{11 / 2}$ & 475915 & 475895 & 2 & $94 \%{ }^{2} \mathrm{H}$ & 0.790 & 0.895 & & $*(4)$ \\
\hline & ${ }^{2} \mathrm{H}_{9 / 2}$ & 475910 & 475937 & 2 & $56 \%{ }^{2} H+11 \% 5 s 5 p\left({ }^{3} P\right) 5 g^{4} F+10 \% 5 s 5 p\left({ }^{3} P\right) 5 g^{2} H+7 \% 5 s 5 p\left({ }^{3} P\right) 5 g{ }^{4} H+6 \% 4 f 5 s\left({ }^{1} F\right) 5 d^{2} H$ & 0.128 & 0.153 & & $*(4)$ \\
\hline \multirow{2}{*}{$5 s^{2} 8 k$} & ${ }^{2} K_{15 / 2}$ & 476369 & 476372 & 1 & $98 \%^{2} \mathrm{~K}$ - & 2.015 & 2.041 & & $*(4)$ \\
\hline & ${ }^{2} K_{13 / 2}$ & 476369 & 476372 & 1 & $98 \%{ }^{2} \mathrm{~K}$ & 2.014 & 2.041 & & $*(4)$ \\
\hline \multirow[t]{8}{*}{$5 s 5 p^{2}$} & ${ }^{4} P_{1 / 2}$ & 92586 & 92674 & 12 & $94 \%{ }^{4} P$ & 10.288 & 14.863 & & (2) \\
\hline & ${ }^{4} P_{3 / 2}$ & 100378 & 100444 & 15 & $99 \%{ }^{4} P$ & 67.957 & 85.780 & & (2) \\
\hline & ${ }^{4} P_{5 / 2}$ & 107205 & 107064 & 16 & $88 \%{ }^{4} P+12 \% 5 s 5 p^{2}{ }^{2} D$ & 11.931 & 13.164 & & (2) \\
\hline & ${ }^{2} D_{3 / 2}$ & 124869.9 & 125034 & 19 & $87 \%{ }^{2} D+6 \% 5 s^{2} 5 d^{2} D$ & 0.574 & 0.612 & & (1) \\
\hline & ${ }^{2} D_{5 / 2}$ & 129229.9 & 129068 & 18 & $80 \%^{2} D+12 \% 5 s 5 p^{2}{ }^{4} P+6 \% 5 s^{2} 5 d^{2} D$ & 1.146 & 1.119 & & (1) \\
\hline & ${ }^{2} P_{1 / 2}$ & 141837.2 & 141660 & 15 & $63 \%{ }^{2} P+32 \% 5 s 5 p^{2}{ }^{2} S$ & 0.091 & 0.130 & $0.14 \pm 0.02$ & (1) \\
\hline & ${ }^{2} S_{1 / 2}$ & 157995.6 & 158078 & 14 & $63 \%{ }^{2} S+34 \% 5 s 5 p^{2}{ }^{2} P$ & 0.111 & 0.159 & $0.145 \pm 0.050$ & (1) \\
\hline & ${ }^{2} P_{3 / 2}$ & 159112.0 & 159192 & 20 & $93 \%^{2} P$ & 0.067 & 0.097 & $0.105 \pm 0.020$ & (1) \\
\hline \multirow[t]{2}{*}{$5 s^{2} 5 d$} & ${ }^{2} D_{3 / 2}$ & 180249.6 & 180237 & 22 & $88 \%{ }^{2} D+6 \% 5 s 5 p^{2}{ }^{2} D$ & 0.049 & 0.132 & $0.115 \pm 0.030$ & (1) \\
\hline & ${ }^{2} D_{5 / 2}$ & 182308.0 & 182319 & 12 & $89 \%^{2} D+5 \% 5 s 5 p^{2}{ }^{2} D$ & 0.060 & 0.163 & $0.12 \pm 0.02$ & (1) \\
\hline $5 s^{2} 6 s$ & ${ }^{2} S_{1 / 2}$ & 223477.8 & 223475 & 7 & $96 \%^{2} S$ & 0.063 & 0.039 & $0.050 \pm 0.005$ & (1) \\
\hline \multirow[t]{2}{*}{$5 s^{2} 6 d$} & ${ }^{2} D_{3 / 2}$ & 338220 & 338385 & 5 & $56 \%^{2} D+31 \% 4 f 5 s\left({ }^{3} F\right) 5 p^{2} D+6 \% 4 f 5 s\left({ }^{1} F\right) 5 p^{2} D$ & 0.109 & 0.304 & & $*(5)$ \\
\hline & ${ }^{2} D_{5 / 2}$ & 339780 & 339609 & 6 & $54 \%^{2} D+36 \% 4 f 5 s\left({ }^{3} F\right) 5 p^{2} D$ & 0.112 & 0.314 & & $*(5)$ \\
\hline $5 s^{2} 7 s$ & ${ }^{2} S_{1 / 2}$ & 352260 & 352260 & 5 & $96 \%^{2} S$ & 0.091 & 0.052 & & $*(5)$ \\
\hline \multirow[t]{2}{*}{$5 s^{2} 5 g$} & ${ }^{2} G_{9 / 2}$ & 376995 & 376981 & 6 & $94 \%{ }^{2} G$ & 0.091 & 0.141 & & $*(4)$ \\
\hline & ${ }^{2} G_{7 / 2}$ & 376995 & 377013 & 6 & $94 \%{ }^{2} G$ & 0.090 & 0.139 & & $*(4)$ \\
\hline \multirow[t]{2}{*}{$5 s^{2} 7 d$} & ${ }^{2} D_{3 / 2}$ & 404159 & 404017 & 7 & $86 \%^{2} D+11 \% 5 s 5 p\left({ }^{1} P\right) 6 p^{2} D$ & 0.506 & 0.244 & & $*(5)$ \\
\hline & ${ }^{2} D_{5 / 2}$ & 404830 & 404972 & 9 & $88 \%{ }^{2} D+7 \% 5 s 5 p\left({ }^{1} P\right) 6 p^{2} D$ & 0.621 & 0.254 & & $*(5)$ \\
\hline $5 s^{2} 8 s$ & ${ }^{2} S_{1 / 2}$ & 413613 & 413616 & 5 & $96 \%{ }^{2} S$ & 0.128 & 0.071 & & $*(5)$ \\
\hline \multirow[t]{2}{*}{$5 s^{2} 6 g$} & ${ }^{2} G_{7 / 2}$ & 426122 & 426020 & 4 & $94 \%^{2} G$ & 0.146 & 0.281 & & $*(4)$ \\
\hline & ${ }^{2} G_{9 / 2}$ & 426227 & 426374 & 4 & $69 \%{ }^{2} G+12 \% 5 s 5 p\left({ }^{3} P\right) 5 f{ }^{4} F+11 \% 5 s 5 p\left({ }^{3} P\right) 5 f{ }^{4} G$ & 0.127 & 0.197 & & $*(4)$ \\
\hline \multirow[t]{2}{*}{$5 s^{2} 8 d$} & ${ }^{2} D_{3 / 2}$ & 442613 & 442594 & 4 & $55 \%{ }^{2} D+27 \% 5 s 5 p\left({ }^{3} P\right) 5 f^{2} D$ & 0.153 & 0.179 & & $*(5)$ \\
\hline & ${ }^{2} D_{5 / 2}$ & 443134 & 442921 & 3 & $91 \%^{2} D$ & 0.424 & 0.362 & & $*(5)$ \\
\hline \multirow[t]{2}{*}{$5 s^{2} 7 i$} & ${ }^{2} I_{13 / 2}$ & 457271 & 457275 & 2 & $97 \%{ }^{2} I$ & 1.018 & 1.050 & & $*(4)$ \\
\hline & ${ }^{2} I_{11 / 2}$ & 457271 & 457273 & 2 & $97 \%^{2} I$ & 1.018 & 1.050 & & $*(4)$ \\
\hline \multirow[t]{2}{*}{$5 s^{2} 7 g$} & ${ }^{2} G_{9 / 2}$ & 458417 & 458418 & 2 & $96 \%{ }^{2} G$ & 0.190 & 0.399 & & New \\
\hline & ${ }^{2} G_{7 / 2}$ & 458417 & 458416 & 2 & $96 \%{ }^{2} G$ & 0.187 & 0.390 & & New \\
\hline \multirow[t]{2}{*}{$5 s^{2} 8 i$} & ${ }^{2} I_{13 / 2}$ & 476195 & 476198 & 1 & $97 \%{ }^{2} I$ & 1.500 & 1.564 & & $*(4)$ \\
\hline & ${ }^{2} I_{11 / 2}$ & 476195 & 476197 & 2 & $97 \%^{2} I$ & 1.499 & 1.564 & & $*(4)$ \\
\hline
\end{tabular}

Notes.

Revised value.

Only components greater than $5 \%$ were used.

Lifetimes calculated for different values of $\alpha$ for the Cd-like $[\mathrm{Kr}] 4 d^{10} 5 s^{2}$ core for transitions of the type $5 s^{2} n l-5 s^{2} n^{\prime} l^{\prime}$, with $r_{c}=1.69 a_{0}$. We used $\alpha=0.993 a_{0}^{3}$ and $r_{c}=0.865 a_{0}$, for transitions involving levels with $5 s$ unfilled orbital. ${ }^{c}$ E. Biémont et al., Eur. Phys. J. D 33, 181-191 (2005).

Levels in square brackets not considered in the least-squares adjustment.

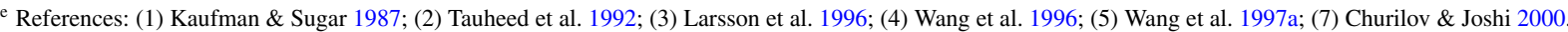


The $5 s^{2} 5 g^{2} G$ levels were classified by Wang et al. (1996) based on three lines, each with double classification. We were able to resolve two pairs of transitions corresponding to the lines previously measured at $1975.2 \AA$ and $1251.4 \AA$. Here, the analysis of those levels counted on a total of 12 lines- -9 more than previously known.

\subsection{Configurations $5 s^{24} f$ and $5 s 4 f 5 p$}

Levels $5 s^{2} 4 f$ were identified by Larsson et al. (1996) and by Wang et al. (1996) based on nine lines measured with an uncertainty in the interval $0.5-1.0 \AA$ and we confirmed all their identifications. Experimental levels of the $5 s 4 f 5 p$ configuration appeared in the analysis of Cs VII (Gayasov \& Joshi 1999), Ba VIII (Churilov et al. 2001), La IX (Gayasov \& Joshi 1998; Churilov \& Joshi 2001), and Cex (Joshi et al. 2001), but they have not been observed in Xe VI and other members of the isoelectronic sequence. This seems to be an important configuration for the future extension of the Xe vi analysis, since its levels are in the interval 258-328 $10^{3} \mathrm{~cm}^{-1}$ (273-343.5 10 $\mathrm{cm}^{-1}$ according to Biémont et al. 2005), between the $5 s^{2} 6 s$ and $5 s^{2} 6 d$ configurations. In fact, the levels $5 s^{2} 6 d$ interact strongly with the $5 s 4 f\left({ }^{3} F\right) 5 p^{2} D$ levels, an effect previously noted by Biémont et al. (2005). From our study of the isoelectronic sequence, we expect the resonant $5 s 4 f 5 p$ lines to lie in the interval 300-400 $\mathrm{A}$.

\subsection{Configurations with $n \geqslant 6$}

The lines at 597.07, 657.81, 709.33, 776.28, and 1165.86 ̊, classified by Reyna Almandos et al. (2001) and reclassified by Saloman (2004) as transitions with $n \geqslant 6$ levels, were not confirmed in the present work. The exception was the line at $687.59 \AA$, reclassified by Saloman (2004) as $5 s 5 p^{22} D_{3 / 2}-$ $5 s^{2} 6 p^{2} P_{3 / 2}$ and confirmed here.

We resolved the line at $2798.1 \pm 0.8 \AA$, identified by Wang et al. (1997a), as the superposition of the $5 s^{2} 6 d^{2} D_{3 / 2}-$ $5 s^{2} 7 p^{2} P_{1 / 2}$ and $5 s^{2} 6 d^{2} D_{5 / 2}-5 s^{2} 7 p^{2} P_{3 / 2}$ transitions, which we measured as two separate lines at $2798.03 \AA$ and $2798.58 \AA$. This measurement allowed us to correct the energy of the level $5 s^{2} 6 d^{2} D_{3 / 2}$ to $338220 \mathrm{~cm}^{-1}$, and to reject the line at $1359.8 \AA$, replacing it by a new line which we observed at $1363.66 \AA$.

The line $2530.6 \AA$ (at $2531.4 \AA$ in vacuum) classified by Wang et al. (1997a) as the $5 s^{2} 7 p^{2} P_{1 / 2}-5 s^{2} 8 s^{2} S_{1 / 2}$ transition, had its wavelength revised to $2530.47 \AA$, and was reclassified here as the $5 s 5 p\left({ }^{1} P\right) 6 s^{2} P_{3 / 2}-5 s^{2} 7 d^{2} D_{5 / 2}$ transition.

We did not confirm any of the four lines previously used to define the $5 s^{2} 7 d$ levels by Wang et al. (1997a), or the line $776.28 \AA ̊$ observed by Reyna Almandos et al. (2001) and later reclassified by Saloman (2004). Instead, we located 16 lines, all but one of which were classified for the first time.

The levels of the configuration $5 s^{2} 7 h$ were first determined by Wang et al. (1996) based on two doubly classified lines. We confirmed their identifications and improved the accuracy of their measurements by resolving the blended line at $1251.4 \AA$ as two lines at 1251.50 and $1253.25 \AA$, and identifying a line at $5151.79 \AA$ for the first time.

The level $5 s^{2} 8 s$ was previously classified by Wang et al. (1997a) on the basis of four transitions to $5 s^{2} 6 p$ and $5 s^{2} 7 p$ levels. We do not confirm any of them. Instead, we found five new lines that redefined the $5 s^{2} 8 s$ level $97 \mathrm{~cm}^{-1}$ below its previous value.

\subsection{Configuration $5 s 5 p 5 d$}

From this point on, we will abbreviate Churilov \& Joshi (2000) to C\&J, and use $s p d$ as shorthand for $5 s 5 p 5 d$ in the level identification.

The first identification of the $5 s 5 p 5 d$ levels was by Sarmiento et al. (1999), but their analysis was entirely rejected by $C \& J$ based on 38 lines. The paper of Reyna Almandos et al. (2001), containing the full list of lines used by Sarmiento et al. (1999), had not been published at the time of the $\mathrm{C} \& \mathrm{~J}$ analysis, and therefore they could not take it into account. Later, Saloman (2004) judged the C\&J analysis to be preferable, and their lines and levels are present in the current version of the Atomic Spectra Database of the National Institute of Standards and Technology (Kramida et al. 2014). Saloman (2004) also reclassified 11 lines from the work of Reyna Almandos et al. (2001), 7 of which were initially identified as transitions with the $5 s 5 p 5 d$ configuration. We rejected all but two of those lines, now classified as indicated in Table 3 where they are marked with the symbol $\dagger$. None of the levels classified by Sarmiento et al. (1999) were confirmed in our analysis.

In the present work, we revised the energy levels of the $5 s 5 p 5 d$ configuration based on 96 lines, 78 of which were classified for the first time. This allowed us to confirm 4, revise 1 , and reject 15 of the levels found by $\mathrm{C} \& \mathrm{~J}$, as well as to classify 22 of the 23 levels of this configuration.

The isoelectronic sequence for the difference between observed and calculated energy levels of $5 s 5 p 5 d$ is shown in Figure 1. Values from C\&J are plotted only if available and visually distinguishable. As we can see, the sequences do not show a very clear regularity, mainly because of the relative change in level positions as a function of the ionization degree. This variation can be attributed to the changes in eigenvector composition, and to the ability of the different coupling schemes to adequately describe the systems as we progress along the isoelectronic sequence.

As is apparent in Table 1, there are strong interactions between the $5 s 5 p 5 d$ and $5 p^{3}$ configurations. Furthermore, the CI integrals corresponding to $5 s 5 p 5 d-5 s^{2} 5 f$ and $5 s 5 p 5 d-$ $5 s 5 p 6 s$ have significant values. These configurations are present in the percentage composition of the $\operatorname{spd}\left({ }^{3} P\right)^{2} F_{5 / 2},{ }^{2} D_{5 / 2}$, ${ }^{2} P_{1 / 2}$, and ${ }^{4} P_{3 / 2}$ and $\operatorname{spd}\left({ }^{1} P\right)^{2} F_{7 / 2},{ }^{2} F_{5 / 2},{ }^{2} D_{3 / 2},{ }^{2} D_{5 / 2},{ }^{2} P_{1 / 2}$, and ${ }^{2} P_{3 / 2}$ energy levels shown in Table 4.

Usually, level classification is performed based on line combinations, line intensity predictions, isoelectronic trends, and the quality of the least-squares fit to experimental levels. However, in this work, we could not always meet all of these criteria simultaneously. In some cases, we had to choose whether to classify levels based on a single strong transition, or based on many lines displaying good wavelength agreement as well as good least-squares fitting. In this regard, the fact that there are no other configurations with unknown levels in the region where $5 s 5 p 5 d$ lies, except the $5 s 5 p 6 s$ which we will discuss below, was considered in our analysis to be a point in favor of the second option.

\subsection{1. $5 s 5 p\left({ }^{3} P\right) 5 d$ Levels}

The isoelectronic trend displayed a sharp edge at Xe vI for the $\operatorname{spd}\left({ }^{3} P\right)^{4} F$ levels, hampering the usage of this resource in our analysis. We corroborated the classification of the level $\operatorname{spd}\left({ }^{3} P\right)^{4} F_{5 / 2}$ by $\mathrm{C} \& \mathrm{~J}$ with the identification of two new lines. However, the line at $616.650 \AA$, classified by $C \& J$ as the $5 s 5 p^{24} P_{3 / 2}-s p d\left({ }^{3} P\right)^{4} F_{5 / 2}$ transition appeared in our 


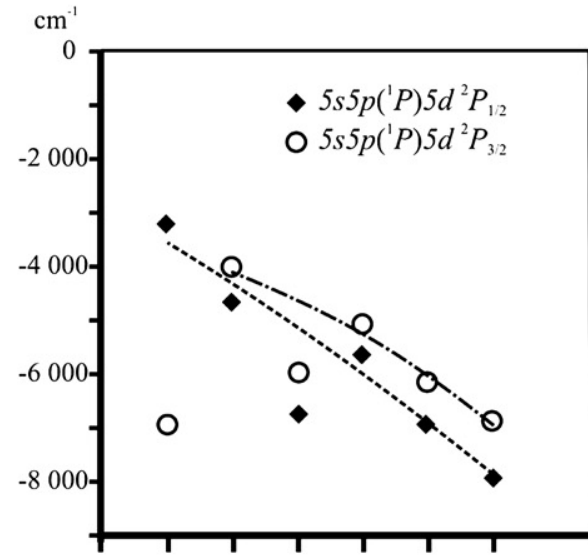

Sb III Te IV I V Xe VI Cs VII Ba VIII
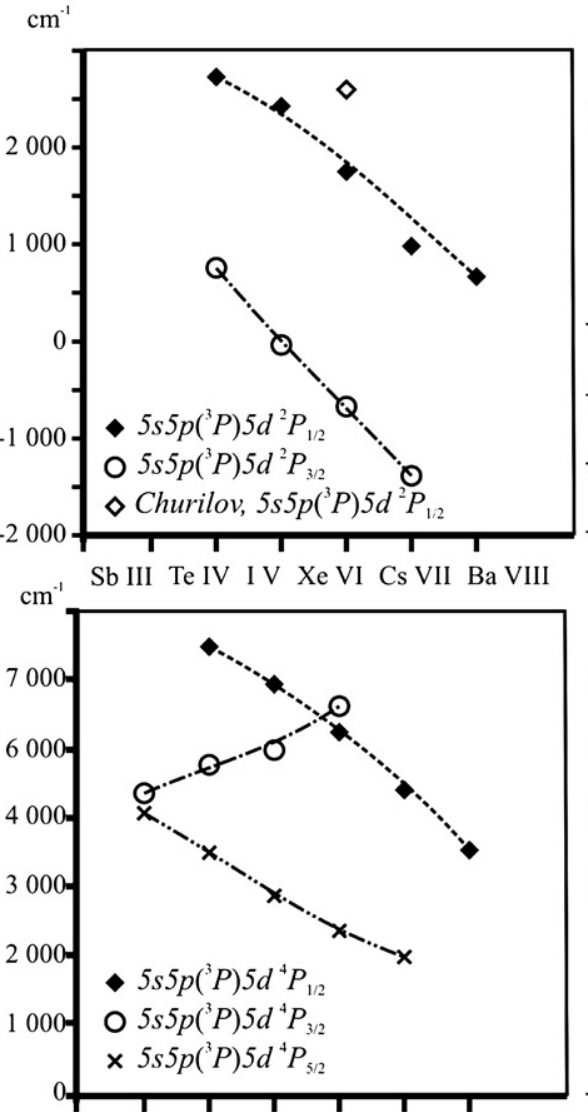

Sb III Te IV I V Xe VI Cs VII Ba VIII

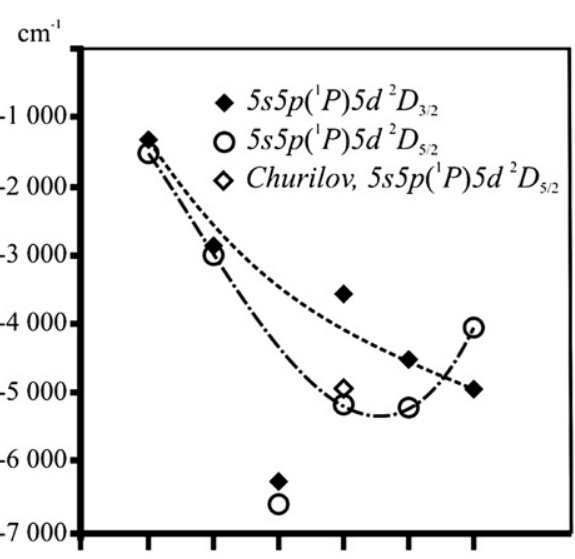

Sb III Te IV I V Xe VI Cs VII Ba VIII

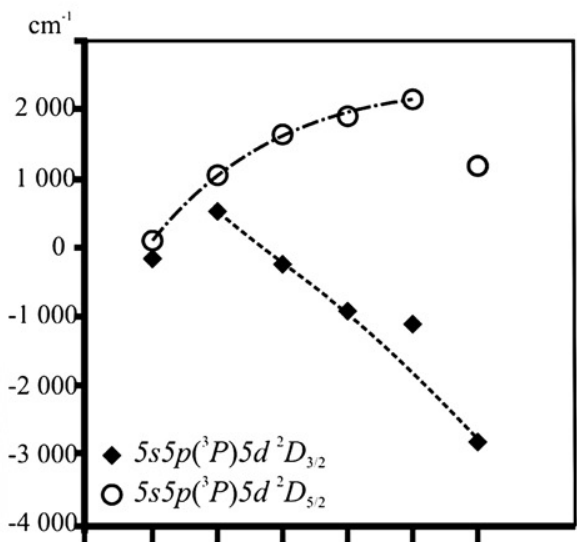

Sb III Te IV I V Xe VI Cs VII Ba VIII

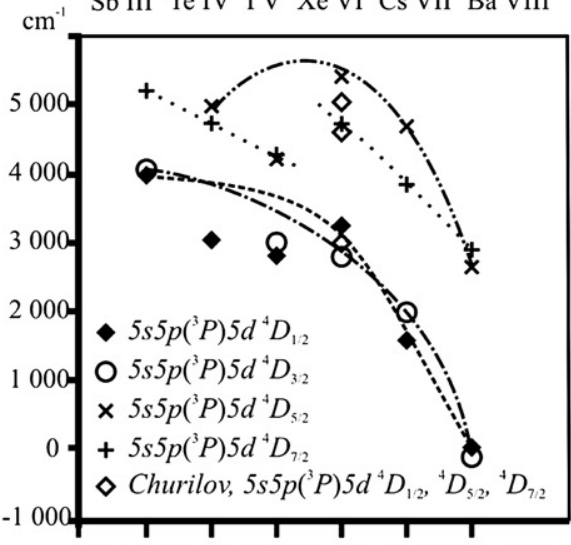

Sb III Te IV I V Xe VI Cs VII Ba VIII

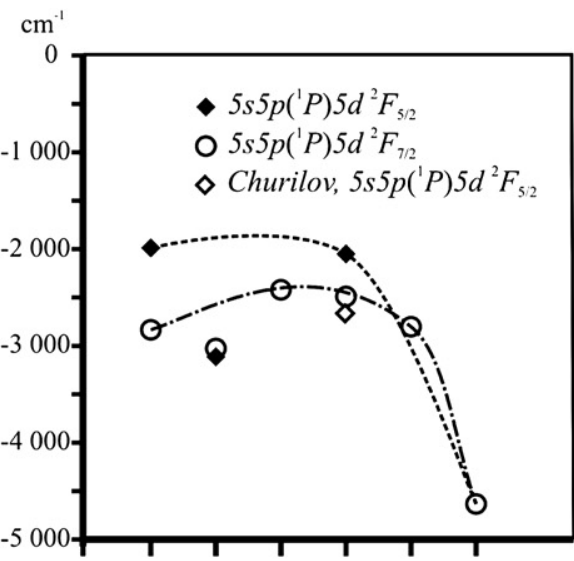

Sb III Te IV I V Xe VI Cs VII Ba VIII

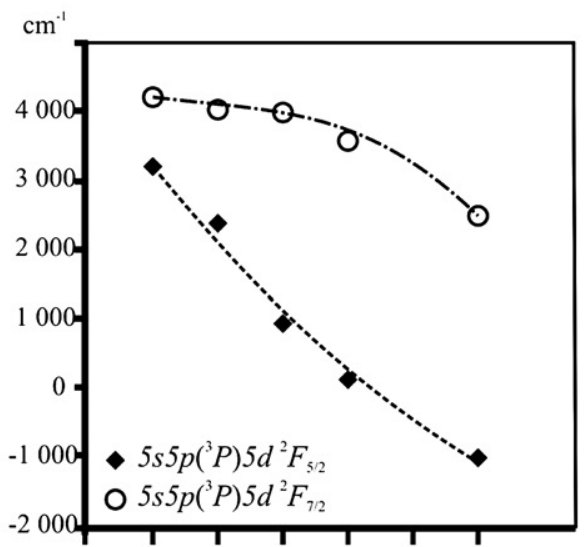

Sb III Te IV IV Xe VI Cs VII Ba VIII

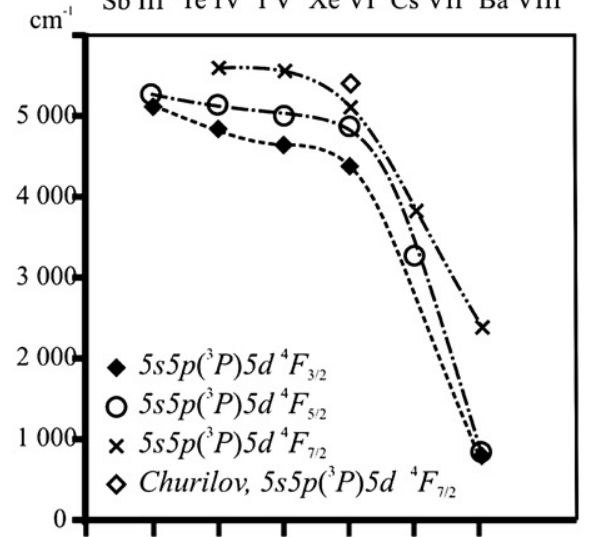

Sb III Te IV I V Xe VI Cs VII Ba VIII

Figure 1. Isoelectronic sequence for the difference between observed and calculated (HFR) energy levels of the $5 s 5 p 5 d$ configuration. Smoothed lines are simply to illustrate trends. Top: $5 s 5 p\left({ }^{1} P\right) 5 d$, (Left) ${ }^{2} P$, (Center) ${ }^{2} D$, (Right) ${ }^{2} F$; center: $5 s 5 p\left({ }^{3} P\right) 5 d$, (Left) ${ }^{2} P$, (Center) ${ }^{2} D$, (Right) ${ }^{2} F$; bottom: $5 s 5 p\left({ }^{3} P\right) 5 d$, (Left) ${ }^{4} P$, (Center) ${ }^{4} D$, (Right) ${ }^{4} F$.

spectrograms as Xe VII or VIII (i.e., it appeared only in experiments where more energy is available than in those where Xe VI usually appears), and was therefore rejected. In addition, we classified the $\operatorname{spd}\left({ }^{3} P\right)^{4} F_{3 / 2}$ level based on five new lines. In the $5 s 5 p 5 d$ configuration, the only level that remains unknown is the $\left({ }^{3} P\right)^{4} F_{9 / 2}$ level, for which our estimated value is $275328 \mathrm{~cm}^{-1}$, as obtained by least-squares fitting. The two lines used by $\mathrm{C} \& \mathrm{~J}$ to identify the $\operatorname{spd}\left({ }^{3} P\right)^{4} F_{7 / 2}$ level appeared in our spectrograms with an unclear ionic assignment. To establish that level, we propose to replace these lines by a set of four new lines that have intensities at different excitation conditions consistent with an Xe VI assignment in our spectrograms.
We rejected all of the $\operatorname{spd}\left({ }^{3} P\right)^{4} D$ levels previously classified by C\&J. In their analysis, all of these but $\operatorname{spd}\left({ }^{3} P\right)^{4} D_{5 / 2}$ were determined on the basis of just 1 transition per level, whereas we could identify a total of 12 new lines. All of the stronger transitions predicted by the theory are present in our spectrograms. For the $J=5 / 2$ level, both C\&J and ourselves identified the same group of three transitions with the $5 s 5 p^{2}$ configuration but with different wavelengths. In this case, our lines produced a better least-squares fit for our set of levels. In addition to the revisions mentioned above, we also rejected the reclassification of the lines 776.28, 971.52, and $637.67 \AA$ originally observed by Reyna Almandos et al. (2001), 

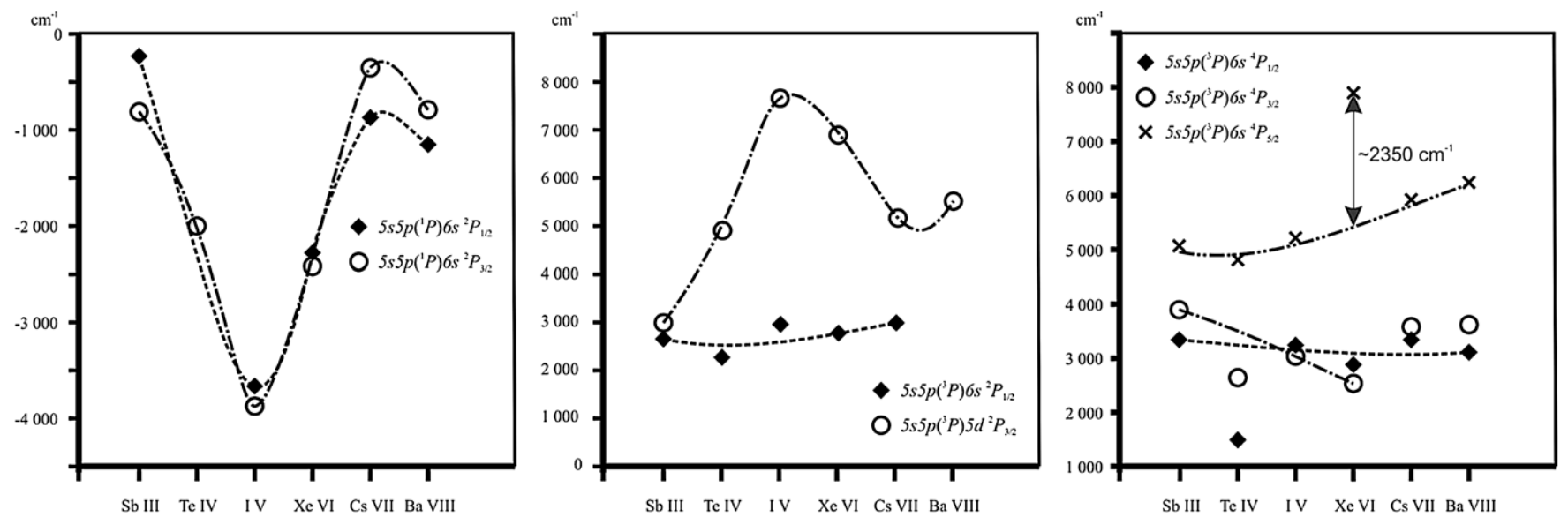

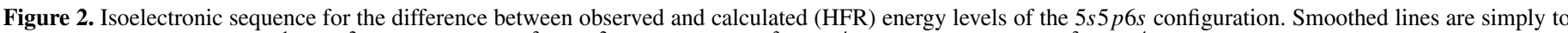

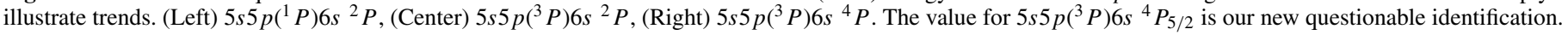

as published by Saloman (2004). Only the line $637.67 \AA$, which was reassigned in this work to the $5 s 5 p^{22} D_{5 / 2}-s p d\left({ }^{3} P\right)^{2} D_{3 / 2}$ transition, remains in our list of wavelengths.

We confirmed the classification of level $\operatorname{spd}\left({ }^{3} P\right)^{4} P_{5 / 2}$ with help of two new lines, but rejected the previous $\operatorname{spd}\left({ }^{3} P\right)^{4} P_{1 / 2}$ classification by $\mathrm{C} \& \mathrm{~J}$. For this level, they used only one line, supposedly corresponding to the highest value of $g A$, for a transition from this level. Our choice led to the identification of a level approximately $100 \mathrm{~cm}^{-1}$ above the previous value, and was based on the fact that our spectrograms showed four new lines that we could identify as transitions to the $5 s 5 p^{2}$ configuration. We were also able to find the $\operatorname{spd}\left({ }^{3} P\right)^{4} P_{3 / 2}$ level based on five new lines, only $60 \mathrm{~cm}^{-1}$ away from the value predicted by $C \& \mathrm{~J}$ in their least-squares calculation. The isoelectronic trend is very smooth for the three levels of this multiplet, as can be seen in Figure 1.

We confirmed the identification by $C \& J$ for the level $\operatorname{spd}\left({ }^{3} P\right)^{2} F_{5 / 2}$, and added five other lines to those previously identified. However, the line at $564.466 \AA$, used by C\&J to determine this level, appears on our plates as an Xe v line. For the other level of the doublet $(J=7 / 2)$, the strong line at $584.761 \AA$ classified by C\&J seems to be an Xe VIII or IX line in our spectrograms. We therefore propose a new energy value for this level based on three newly observed lines. In both cases, the isoelectronic sequence shows a smooth trend and our values are very close to those obtained by $\mathrm{C} \& \mathrm{~J}$.

We agreed with the classification for $\operatorname{spd}\left({ }^{3} P\right)^{2} D_{5 / 2}$ proposed by $\mathbf{C} \& \mathbf{J}$ based on only one line, and improved its energy value with the observation of three new lines. In addition, we reclassified the line at $598.211 \AA$, previously classified by $\mathrm{C} \& \mathrm{~J}$ as $5 s 5 p^{22} P_{3 / 2}-s p d\left({ }^{1} P\right)^{2} P_{3 / 2}$, as corresponding to the $5 s 5 p^{22} D_{3 / 2}-s p d\left({ }^{3} P\right)^{2} D_{5 / 2}$ transition. One reclassified line from Reyna Almandos et al. (2001) and six new lines support our analysis of the $\operatorname{spd}\left({ }^{3} P\right)^{2} D_{3 / 2}$ level, on the basis of which we rejected the choice of $\mathrm{C} \& \mathrm{~J}$ for this level, which relied on a single transition.

The new energy values that we proposed for the $\operatorname{spd}\left({ }^{3} P\right)^{2} P$ levels to replace the values found by $C \& J$ are based on a set of 13 lines, some of which are from the reclassified level $\operatorname{spd}\left({ }^{3} P\right)^{2} P_{1 / 2}$ previously identified (Reyna Almandos et al. 2001) as $5 s 5 p\left({ }^{3} P\right) 6 s^{4} P_{3 / 2}$, and some of which are new. Thus, our identifications are much more reliable than the previous ones by $\mathrm{C} \& \mathrm{~J}$, which were based on just one transition per level. The same conclusion emerges from our analysis of $\operatorname{spd}\left({ }^{3} P\right)^{2} P_{1 / 2}$ isoelectronic trend, as we can see in Figure 1.

\subsection{2. $5 s 5 p\left({ }^{1} P\right) 5 d$ Levels}

We found a second transition with the $\operatorname{spd}\left({ }^{1} P\right)^{2} F_{7 / 2}$ level proposed by $\mathrm{C} \& \mathrm{~J}$, confirming their identification. We also found four new lines that set the $\operatorname{spd}\left({ }^{1} P\right)^{2} F_{5 / 2}$ level at $316868 \mathrm{~cm}^{-1}$, replacing the level found by $\mathrm{C} \& \mathrm{~J}$ based on only one line.

We did not observe the pair of lines used by $C \& J$ to define the spd 323343 3/2 level (notation from C\&J), leaving no other alternative for us but to reject their classification. In its place, we identified four new Xe VI lines that shift the level to less than $30 \mathrm{~cm}^{-1}$ above the previous value for $\operatorname{spd}\left({ }^{1} P\right)^{2} D_{3 / 2}$.

While we did not identify the line at $705.528 \AA$ as Xe VI, we considered another set of lines for $\operatorname{spd}\left({ }^{1} P\right)^{2} D_{5 / 2}$ from the reclassification of the level $s p d\left({ }^{1} P\right)^{2} D_{3 / 2}$ previously classified by Reyna Almandos et al. (2001), in addition to two new transitions with the $5 s 5 p^{2}$ and $5 s^{2} 7 d$ configurations, moving the $\operatorname{spd}\left({ }^{1} P\right)^{2} D_{5 / 2}$ level to $323772 \mathrm{~cm}^{-1}$.

The strong line at $606.310 \AA$, previously classified by $C \& \mathrm{~J}$ as $5 s 5 p^{22} P_{3 / 2}-s p d\left({ }^{1} P\right)^{2} D_{5 / 2}$, was reclassified in the present work as $5 s 5 p^{22} P_{3 / 2}-s p d\left({ }^{1} P\right)^{2} P_{1 / 2}$. The new value for the $\operatorname{spd}\left({ }^{1} P\right)^{2} P_{1 / 2}$ energy level is based on two lines. We did not find the lines at 598.211 and $694.590 \AA$ in our spectrograms, and we identified the line at $594.246 \AA$ as an Xe VIII line. Therefore, we reclassified the $\operatorname{spd}\left({ }^{1} P\right)^{2} P_{3 / 2}$ level based on six new lines, displacing the energy level by $56 \mathrm{~cm}^{-1}$ from the value predicted by C\&J. Finally, we reclassified the $\operatorname{spd}\left({ }^{1} P\right)^{2} D_{3 / 2}$ based on four lines instead of the two lines previously used by C\&J.

\subsection{Configuration $5 s 5 p 6 s$}

Prior to this work, only three levels, determined from six lines, were known for the $5 s 5 p 6 s$ configuration (Sarmiento et al. 1999; Reyna Almandos et al. 2001). However, we had to reject all of these values. We present a new analysis for the $5 s 5 p 6 s$ configuration based on 19 lines. All but one of these are new classifications. The isoelectronic trend for these levels is shown in Figure 2, in which we see the same discontinuities as found in the $5 s 5 p 5 d$ configuration, and for the same reasons, as explained in Section 5.4. For the $5 s 5 p 6 s$ configuration, there is significant mixing for the ${ }^{2} P_{1 / 2}$ and ${ }^{2} P_{3 / 2}$ energy levels between the $5 s 5 p\left({ }^{1} P\right) 6 s$ and $5 s^{2} 7 p$ configurations, as shown in Table 4 . 
This configuration exhibits strong mixing with $5 s 5 p 5 d$, especially between the $5 s 5 p\left({ }^{3} P\right) 6 s^{4} P_{3 / 2}$ and $s p d\left({ }^{1} P\right)^{2} D_{3 / 2}$ levels at $321637 \mathrm{~cm}^{-1}$ and $323370 \mathrm{~cm}^{-1}$, respectively. In both cases, the $5 s 5 p\left({ }^{3} P\right) 6 s^{4} P_{3 / 2}$ component is dominant, and so the classification could actually be reversed.

We found all but the $5 s 5 p\left({ }^{3} P\right) 6 s^{4} P_{5 / 2}$ level of the $5 s 5 p 6 s$ configuration. For this level, we did find a possible classification, but doubts remain about it. On the one hand, the experimental value found, $337032 \mathrm{~cm}^{-1}$, agrees well with the fitted value from the least-squares calculation, differing by only $30 \mathrm{~cm}^{-1}$. However, on the other hand, this energy value displaces Xe vi from the isoelectronic trend by about $2350 \mathrm{~cm}^{-1}$ - too far off to consider this classification as reliable. For this reason, we left this level and the corresponding lines (at 435.08 and $562.05 \AA$ ) out of our tables.

\section{DISCUSSION}

The Xe VI analysis has been difficult because of the strong mixing of level composition and the non-smooth behavior of the structure, which together result in changes in level positions and composition along the isoelectronic sequence. In this work, nine lines in the visible region and a number of ultraviolet lines were identified for the first time, which can be helpful to support future analysis of spectra of cosmic objects. All Xe vi lines recently observed in the spectrum of the hot DO-type white dwarf RE 0503-289 by Werner et al. (2012) were confirmed. This work has doubled the spectral information concerning this ion.

This research was supported by the Consejo Nacional de Investigaciones Científicas y Técnicas (CONICET), Argentina, by the Coordenação de Aperfeiçoamento de Pessoal de Nível Superior (CAPES), and by the Conselho Nacional de Pesquisa (CNPq), Brazil. Support of the Comisión de Investigaciones Científicas de la Província de Buenos Aires (CIC), where J. G. R. A. and M. R. are researchers, is also gratefully acknowledged.

\section{REFERENCES}

Biémont, É., Buchard, V., Garnir, H.-P., Lefèbvre, P.-H., \& Quinet, P. 2005, EPJD, 33, 181

Biémont, É., Fischer, C. F., Godefroid, M. R., Palmeri, P., \& Quinet, P. 2000a, PhRvA, 62, 032512

Biémont, É., Pinnington, E. H., Quinet, P., \& Zeippen, C. J. 2000b, PhyS, 61,567

Churilov, S. S., \& Joshi, Y. N. 2000, PhyS, 62, 358

Churilov, S. S., \& Joshi, Y. N. 2001, PhyS, 64, 34

Churilov, S. S., Joshi, Y. N., \& Gayasov, R. J. 2001, JOSAB, 18, 113
Cowan, R. D. 1981, The Theory of Atomic Structure Spectra (Berkeley, CA: Univ. California Press)

Cowley, C. R., Hubrig, S., Palmeri,, et al. 2010, MNRAS, 405, 1271

Curtis, L. J. 2003, Atomic Structure Lifetimes: A Conceptual Approach (Berkeley, CA: Univ. California Press)

Dworetsky, M. M., Persaud, J. L., \& Patel, K. 2008, MNRAS, 385, 1523

Edlén, B. 1964, Atomic Spectra, Handbuch der Physik, Vol. 27 (Berlin: Springer), 80

Edlén, B. 1966, Metro, 2, 71

Fawcett, B. C., \& Bromage, G. E. 1980, JPhB, 13, 2711

Fawcett, B. C., Jones, B. B., \& Wilson, R. 1961, PPS, 78, 1223

Fraga, S., \& Muszyńska, J. 1981, Atoms in External Fields (Amsterdam: Elsevier Scientific)

Gallardo, M., Raineri, M., Reyna Almandos, J. G., et al. 1995, PhyS, 51, 737

García-Rojas, J., Peña, M., Morisset, C., Mesa-Delgado, A., \& Ruiz, M. T. 2012, A\&A, 538, 84

Gayasov, R., \& Joshi, Y. N. 1998, JOSAB, 15, 2614

Gayasov, R., \& Joshi, Y. N. 1999, PhyS, 60, 312

Hansen, J. E., \& Persson, W. 1987, PhyS, 36, 602

Joshi, Y. N., Ryabtsev, A. N., \& Churilov, S. S. 2001, PhyS, 64, 326

Kaufman, V., \& Sugar, J. 1987, JOSAB, 4, 1924

Kelly, R. L. 1987, JPCRD, 16, 1

Kernahan, J. A., Pinnington, E. H., O’Neill, J. A., Bahr, J. L., \& Donnelly, K. E. 1980, JOSA, 70, 1126

Kramida, A., Ralchenko, Yu., Reader, J., \& NIST ASD Team., 2014, NIST Atomic Spectra Database (version 5.2), http://physics.nist.gov/asd (Gaithersburg, MD: National Institute of Standards and Technology)

Larsson, M. O., Gonzalez, A. M., Hallin, R., et al. 1996, PhyS, 53, 317

Migdalek, J., \& Baylis, W. E. 1978, JPhB, 11, L497

Otsuka, M., \& Tajitsu, A. 2013, ApJ, 778, 146

Otsuka, M., Tajitsu, A., Hyung, S., \& Izumiura, H. 2010, ApJ, 723, 658

Pagan, C. J. B., Cavalcanti, G. H., Jupén, C., \& Trigueiros, A. G. 2011, ApJS, 196,19

Péquignot, D., \& Baluteau, J.-P. 1994, A\&A, 283, 593

Persson, W., Wahlström, C.-G., Bertuccelli, G., et al. 1988, PhyS, 38, 347

Quinet, P., Palmeri, P., Biémont, É., et al. 1999, MNRAS, 307, 934

Quinet, P., Palmeri, P., Biémont, É., et al. 2002, JAIIC, 344, 255

Raineri, M., Gallardo, M., Pagan, C. J. B., Trigueiros, A. G., \& Reyna Almandos, J. 2012, JQSRT, 113, 1612

Reyna Almandos, J., Bredice, F., Raineri, M., \& Gallardo, M. 2009, PhyS, T134, 014018

Reyna Almandos, J., Sarmiento, R., Raineri, M., Bredice, F., \& Gallardo, M. 2001, JQSRT, 70, 189

Saloman, E. B. 2004, JPCRD, 33, 765

Sarmiento, R., Reyna Almandos, J. G., Raineri, M., \& Gallardo, M. 1999, JPhB, 32,2853

Sharpee, B., Zhang, Y., Williams, R., et al. 2007, ApJ, 659, 1265

Sterling, N. C., Dinerstein, H. L., Hwang, S., et al. 2009, PASA, 26, 339

Tauheed, A., Joshi, Y. N., \& Pinnington, E. H. 1992, JPhB, 25, L561

Tauheed, A., Joshi, Y. N., \& Pinnington, E. H. 1998, JPhB, 31, 393

Tauheed, A., Joshi, Y. N., \& Rana, T. 2000, PhyS, 61, 696

Tauheed, A., Zafaran, A. F., \& Joshi, Y. N. 1999, JPhB, 32, 2917

Valero, F. P. J. 1968, JOSA, 58, 1048

Wang, M., Arnesen, A., Hallin, R., et al. 1997a, JOSAB, 14, 3277

Wang, M., Larsson, M. O., \& Arnesen, A. 1996, JOSAB, 13, 2715

Wang, M., Larsson, M. O., Arnesen, A., et al. (Erratum) 1997b, JOSAB, 14, 1515

Werner, K., Rauch, T., Ringat, E., \& Kruk, J. W. 2012, ApJL, 753, L7 